\title{
Targeting STAT3 in Cancer Immunotherapy
}

\author{
Sailan Zou ${ }^{1+}$, Qiyu Tong ${ }^{1 \dagger}$, Bowen Liu², Wei Huang ${ }^{3}$, Yan Tian ${ }^{1 *}$ and Xianghui Fu ${ }^{1 *}$ (D)
}

\begin{abstract}
As a point of convergence for numerous oncogenic signaling pathways, signal transducer and activator of transcription 3 (STAT3) is central in regulating the anti-tumor immune response. STAT3 is broadly hyperactivated both in cancer and non-cancerous cells within the tumor ecosystem and plays important roles in inhibiting the expression of crucial immune activation regulators and promoting the production of immunosuppressive factors. Therefore, targeting the STAT3 signaling pathway has emerged as a promising therapeutic strategy for numerous cancers. In this review, we outline the importance of STAT3 signaling pathway in tumorigenesis and its immune regulation, and highlight the current status for the development of STAT3-targeting therapeutic approaches. We also summarize and discuss recent advances in STAT3-based combination immunotherapy in detail. These endeavors provide new insights into the translational application of STAT3 in cancer and may contribute to the promotion of more effective treatments toward malignancies.
\end{abstract}

Keywords: STAT3, Cancer, Immunosuppression, Immunotherapy, Immune checkpoint blockade, CAR-T

\section{Introduction}

Dysregulation of immune checkpoints is a protective mechanism used by a number of malignancies to escape from the immune surveillance allowing for cancer development [1]. This has inspired the idea of boosting the host immune response as an anti-cancer therapy. Indeed, the blockage of immune checkpoints, including programmed cell death protein 1 (PD-1), programmed cell death 1 ligand 1 (PD-L1) and cytotoxic T-lymphocyte-associated protein 4 (CTLA-4), improves clinical outcomes in subsets of patients with cancers previously considered to be essentially untreatable [2-4]. In order to expand the array of treatable cancers as well as increase the number of patients that respond to the therapy, novel therapeutic targets and new molecules/strategies should be urgently

\footnotetext{
*Correspondence: tyfxh@163.com; xfu@scu.edu.cn

${ }^{+}$Sailan Zou and Qiyu Tong are co-first author.

'Division of Endocrinology and Metabolism, National Clinical Research Center for Geriatrics, State Key Laboratory of Biotherapy and Cancer Center, West China Hospital, Sichuan University and Collaborative Innovation Center of Biotherapy, Chengdu 610041, Sichuan, China

Full list of author information is available at the end of the article
}

identified and developed for immunotherapy appropriate for the clinical use.

The signal transducer and activator of transcription (STAT) proteins are a family of cytoplasmic transcription factors which share an overall general structure, organized into functional modular domains. The mammalian STAT family comprises STAT1, STAT2, STAT3, STAT4, STAT5a, STAT5b and STAT6 that mediate multiple intracellular signaling pathways [5]. Among them, STAT3 is involved in numerous biological processes including cell proliferation, survival, differentiation, and angiogenesis $[6$, 7]. In normal cells, transient activation of STAT3 (predominantly by phosphorylation) transmits transcriptional signals from cytokines and growth factor receptors at the plasma membrane to the nucleus [5]. In contrast, STAT3 becomes hyperactivated in the majority of human cancers and is generally associated with poor clinical prognosis [8]. Therefore, it is not surprising that STAT3 signaling pathway has long been recognized as a potential therapeutic target for cancer therapy owing to their roles in tumor formation, metastasis and drug resistance [9-12]. Moreover, accumulating evidence reveals that STAT3 hyperactivation can mediate tumor-induced immunosuppression at

(c) The Author(s). 2020 Open Access This article is licensed under a Creative Commons Attribution 4.0 International License, which permits use, sharing, adaptation, distribution and reproduction in any medium or format, as long as you give appropriate credit to the original author(s) and the source, provide a link to the Creative Commons licence, and indicate if changes were made. The images or other third party material in this article are included in the article's Creative Commons licence, unless indicated otherwise in a credit line to the material. If material is not included in the article's Creative Commons licence and your intended use is not permitted by statutory regulation or exceeds the permitted use, you will need to obtain permission directly from the copyright holder. To view a copy of this licence, visit http://creativecommons.org/licenses/by/4.0/. The Creative Commons Public Domain Dedication waiver (http://creativecommons.org/publicdomain/zero/1.0/) applies to the data made available in this article, unless otherwise stated in a credit line to the data. 
many levels $[13,14]$. Given the similarities between tumorigenesis and STAT3-dependent immunity, new therapeutic strategies that target STAT3 signaling pathway may open up new avenues for long-lasting and multilayered tumor control.

This review outlines the role of the STAT3 pathway in tumor immunity, summarizes the recent progress in STAT3-centered anti-cancer approaches, and highlights future directions for the clinical immunotherapy.

\section{The STAT3 signaling pathway}

STAT3 is a protein consisting of 770 amino acids and characterized by the presence of 6 functionally conserved domains, including the amino-terminal domain (NH2), the coiled-coil domain (CCD), the DNA-binding domain (DBD), the linker domain, the SRC homology 2 (SH2) domain, and the carboxyl-terminal transactivation domain (TAD) (Fig. 1a). Among them, SH2 is the most highly conserved STAT domain and plays a crucial role in signaling via binding to specific phosphotyrosine motifs [15]. In an unstimulated cell, STAT3 is tightly regulated by negative modulators to maintain an inactive state in the cytoplasm. These modulators include members of the protein inhibitor of activated STAT (PIAS), suppressor of cytokine signaling (SOCS) families, protein tyrosine phosphatases (SHP1, SHP2, PTPN1, PTPN2 PTPRD, PTPRT and DUSP22), and ubiquitin enzymes [8]. In response to stimuli, STAT3 becomes activated mainly by direct phosphorylation at tyrosine (705) and serine (727) residues induced by its upstream ligands including Janus kinases (JAKs), tyrosine kinases, cytokines and several non-receptor tyrosine kinases such as SRC and $\mathrm{ABL}$; the phosphorylation induces dimerization of STAT3 proteins followed by nuclear translocation, DNA binding, and eventually execution of their nuclear functions [15].

Beyond phosphorylation, other posttranslational modifications (i.e. acetylation, methylation, and sumoylation) can also regulate STAT3 transcriptional activity through altering STAT3 phosphorylation, and thus add another layer of complexity for STAT3 hyperphosphorylation in cancers (Fig. 1b). For instance, acetylation at several lysine residues within both the $\mathrm{NH} 2$ and $\mathrm{SH} 2$ domains, primarily mediated by the $\mathrm{CBP} / \mathrm{p} 300$ acetyltransferase, can enhance STAT3 transactivating potential, which is associated with increased dimer stabilization, tyrosine 705 phosphorylation, nuclear translocation, and localized histone hyperacetylation of target promoters [16]. In contrast, deacetylation by several deacetylases, such as HADC1-3, SIRT1 and Loxl3, inhibits transcription of STAT3 targets [17-19]. The dynamic balance of acetylation and deacetylation plays a role in STAT3 activation and is involved in various cellular events. Similarly, methylation and sumoylation are also emerging as important regulatory mechanisms for STAT3 activity. SMYD2-dependent methylation of STAT3 contributes to the hyperphosphorylation of STAT3, whereas EZH2and SET9-dependent dimethylation of STAT3 inhibits the activity of DNA-bound STAT3 dimers [20-22]. Sumoylation at the lysine 451 of STAT3 by SUMO2/3 can promote its interaction to the nuclear phosphatase TC45, thereby restraining phosphorylated STAT3 in the nucleus, while de-sumoylation by SENP3 leads to the hyperphosphorylation of STAT3 [23]. However, androgen receptor degradation enhancer ASC-J9 can inhibit the STAT3 phosphorylation via inducing the sumoylation of STAT3 at lysine 679 [24].

Additionally, increasing evidence suggests that noncoding RNAs (ncRNAs) can directly or indirectly modulate STAT3 activity (Fig. 1c). As the most extensively studied ncRNAs, numerous microRNAs (miRNAs) have been shown to target STAT3 directly and certain components of STAT3 signaling pathway (IL-6, JAK2, SOCS1, PIAS3, etc.), thereby modulating STAT3 expression and activation [8, 25-29]. For instance, miR-125b$5 p$ can directly target STAT3 and inhibit its expression [27], while miR-218 indirectly suppresses STAT3 activation by targeting IL-6 receptor and JAK3 [28]. More recently, it has shown that exosome-mediated transfer of certain miRNAs, such as miR-193a-3p, miR-210-3p and miR-5100, can promote metastasis of lung cancer by enhancing STAT3 activity [29], although the molecular mechanisms await further investigation. Likewise, long non-coding RNAs (lncRNAs) can modulate the expression and activation of STAT3 directly or indirectly through multiple mechanisms [30-33]. For instance, lincRNA-p21 can inhibit the STAT3 transcriptional activity via directly binding to STAT3 [30]. Lnc-BM can bind to the JH2 domain of JAK2, which increases JAK2 activation, and thus indirectly enhances activity of STAT3 [32]. FLANC, a novel primate-specific IncRNA, has shown to upregulate and prolong the half-life of phosphorylated STAT3, but not total STAT3, albeit the underlying mechanism remains unknown [34]. Intriguingly, a recent study revealed that LINC00908encoded polypeptide ASRPS can directly bind to the CCD domain of STAT3, and thus reduce STAT3 phosphorylation [35]. In general, circular RNAs (circRNAs) can modulate gene expression by acting as sponges of endogenous miRNAs. It has shown that circ-HIPK3, circ_0076305 and circ-STAT3 positively modulate STAT3 signaling by sponging miR-124-3p, miR-296-5p, and $\mathrm{miR}-29 \mathrm{a} / \mathrm{b} / \mathrm{c}-3 \mathrm{p}$, respectively [36-38]. In parallel, STAT3 has a capacity of regulating ncRNAs directly or indirectly. The regulation of miR-21 by STAT3 has been extensively studied. On the one hand, STAT3 can directly regulate miR-21 transcription in myeloma cells by binding to its upstream enhancer region [39]. On the 
a

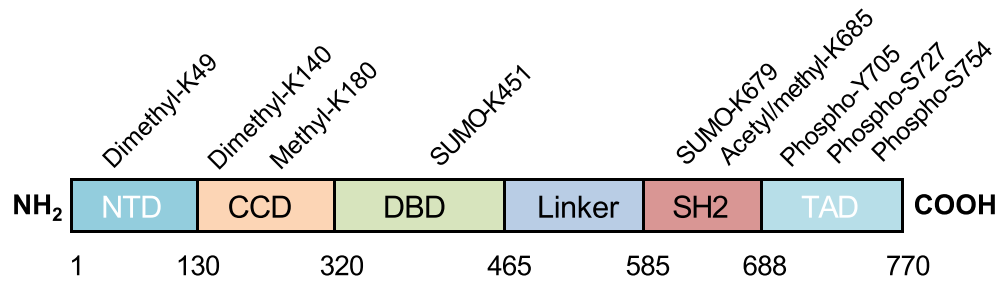

b
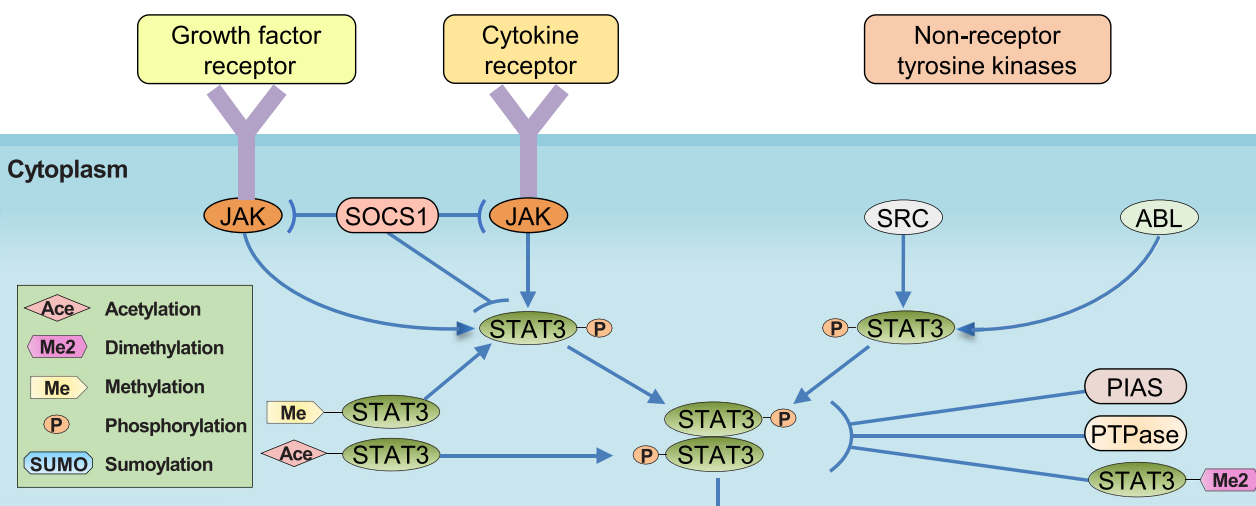

Nucleus $\quad$ SUMO-STAT3 $($ STAT3-P
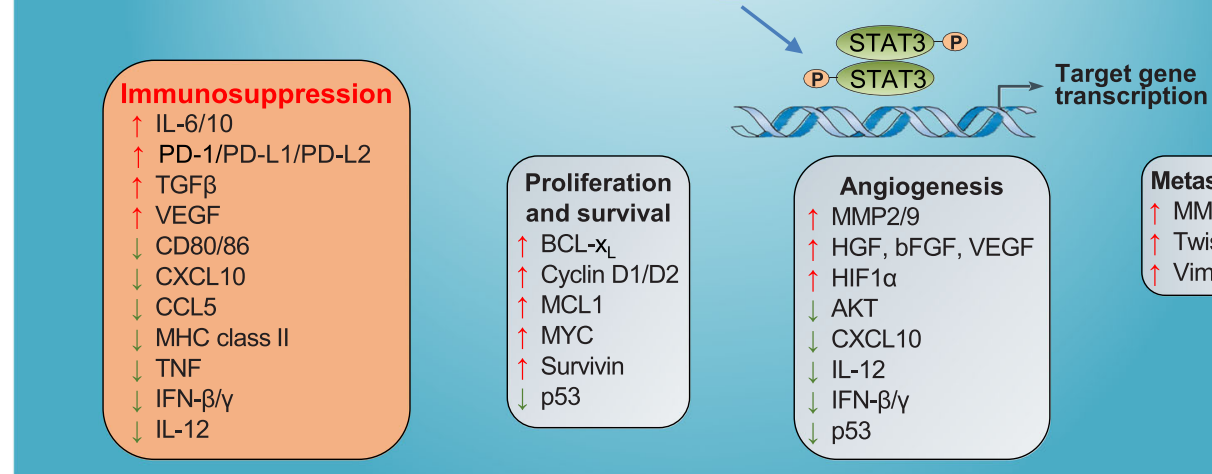

transcription

C

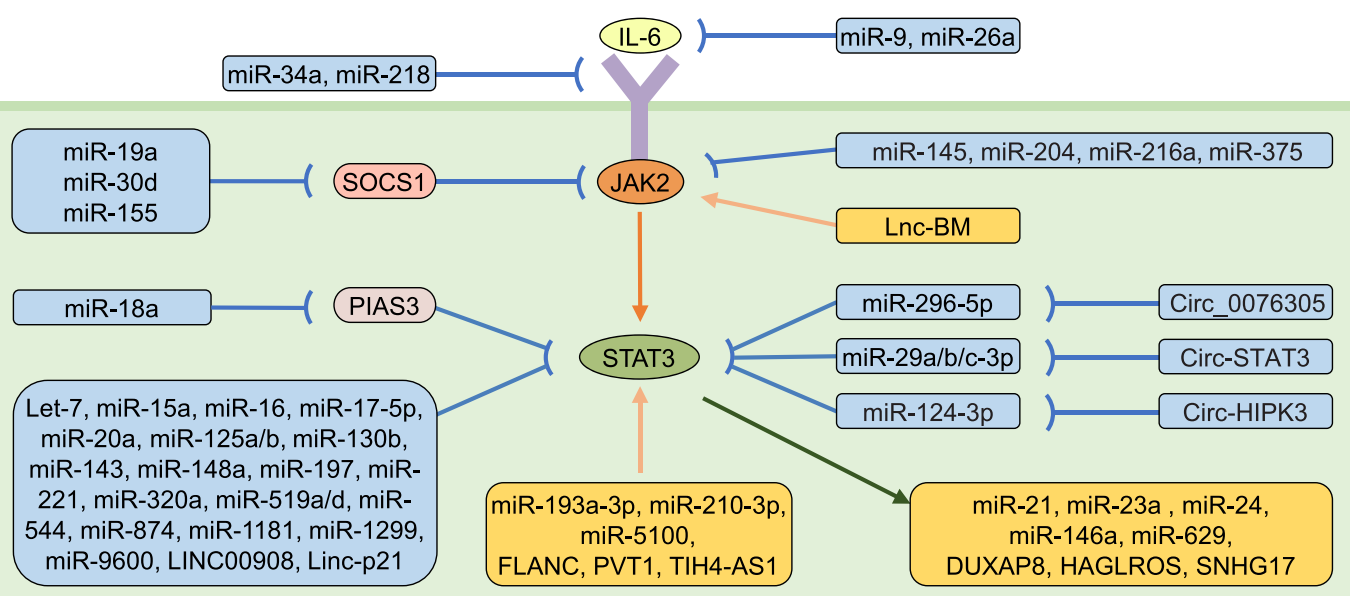

Fig. 1 (See legend on next page.) 
(See figure on previous page.)

Fig. 1 The domain structure and signaling pathway of STAT3. a Schematic domain structure of STAT3. STAT3 is characterized by the presence of six different functional domains, including an amino-terminal domain (NTD) for cooperative binding of STAT proteins to multiple consensus DNA sites, a coiled-coil domain (CCD) for recruitment of STAT3 to the receptor as well as subsequent phosphorylation, dimerization and nuclear translocation, a DNA-binding domain (DBD) for recognizing and binding to a specific consensus DNA sequence, a linker domain for connecting the DBD with the SRC homology 2 (SH2) domain, a SH2 domain for recruitment and activation as well as dimerization of the STAT3 molecule by interacting with phosphorylated tyrosine residues in the opposing subunit, and a carboxyl-terminal transactivation domain (TAD). b STAT3 signaling pathway. STAT3 is activated by upstream growth factor kinases and cytokine receptors. Non-receptor tyrosine kinases such as SRC and ABL can also lead to constitutive activation of STAT3. Phosphorylated STAT3 dimerizes and translocates to nucleus, which causes the transcription of target genes including immunosuppression, angiogenesis, metastasis, proliferation and survival. The signaling pathway can be inhibited by SOCS proteins, PIAS proteins, and protein tyrosine phosphatases (PTPases), etc. c Interplay between noncoding RNAs and STAT3 signaling pathway. On the one hand, miRNAs and IncRNAs can regulate STAT3 activation through not only directly targeting STAT3, but also targeting the components of the STAT3 signaling pathway, such as IL-6, JAK2, SOCS1 and PIAS3; CircRNAs usually regulate STAT3 by acting as sponges for miRNAs. On the other hand, STAT3 is able to regulate miRNAs and IncRNAs expression in many ways.

other hand, STAT3 can upregulate miR-21 through increasing IL-6 expression [40]. The regulatory effect of STAT3 in lncRNAs is also emerging. STAT3 can directly bind to the promoter region of certain lncRNAs, such as SNHG17, DUXAP8 and HAGLROS, and thus contributes to their overexpression in cancers [41-43].

As described above, STAT3 activity can be influenced by many factors such as numerous post-translational modifications and multiple ncRNAs regulation. These complexities, together with the fact that the STAT3 signaling pathway is responsive to a great variety of cellular stresses and stimuli [44], pose difficulties in our understanding of abnormal hyperactivated STAT3 in cancers. Future investigations delineating the regulatory network of STAT3 will likely facilitate the clinical translation of STAT3-based therapies for human malignancy.

\section{STAT3-driven tumor immunosuppression}

The tumor microenvironment (TME) is a highly complex and heterogenous ecosystem consisting tumorinfiltrating immune cells, cancer-associated fibroblasts (CAFs), smooth muscle cells, endothelial cells, and the tumor cells $[45,46]$. It is becoming increasingly evident that TME can promote the progression of cancer and mediate therapeutic resistance, particularly against cancer immunotherapy $[47,48]$. Gathered evidence suggests that STAT3 becomes hyperactivated not only in cancer cells themselves but also in immune cells and CAFs within the TME [13, 49-52]. The hyperactivation of STAT3 in TME compartments might have a significant impact on anti-tumor immunity through various mechanisms (described below in more detail).

\section{STAT3 in tumor cells}

In tumor cells per se, hyperactivated STAT3 decreases the expression of immune-stimulating factors including interferons (IFNs), pro-inflammatory cytokines (IL-12, TNF- $\alpha$ ) and chemokines (CCL5, CXCL10), while increases the expression of certain cytokines and growth factors (IL-6, IL-10, TGF $\beta$, and VEGF), thereby exerting profound immune effects (Fig. 1b)
[53]. For instance, STAT3 can suppress the secretion of type 1 IFNs (IFN-Is) and IFN-I-responsive genes via multiple actions, such as attenuating the activation of IFN-I signaling, reducing the expression of ISGF3 components, and impairing the potential of ISGF3 transactivation $[54,55]$.

In tumor cells, STAT3 often interacts with other signaling pathways, such as NF- $\mathrm{kB}$, to confer robustness for tumor progression $[44,56,57]$. NF- $\kappa B$ signaling is of importance for both inflammation-induced carcinogenesis and anti-tumor immunity [57]. NF- $\mathrm{B}$ (especially RELA) can upregulate a spectrum of targets involved in chronic inflammation and cancer initiation such as cyclooxygenase 2, IL-6, IL-23, and IL$1 \beta$ [44]. Several layers of STAT3-NF-kB crosstalk have been identified thus far: (1) both NF- $\mathrm{kB}$ and STAT3 are frequently activated in the same tumor cells and TME-associated infiltrating immune cells, and share a wide range of common targets that participate in cell proliferation, metastasis, anti-apoptosis, and angiogenesis [56]; (2) STAT3 can prolong nuclear retention of RELA through p300-mediated acetylation, leading to the persistent activation of NF- $\mathrm{B}$ [58]; (3) many cytokines (i.e. IL-6) can in turn simultaneously activate STAT3 and NF-kB [57]; (4) it has recently been demonstrated that NF- $\mathrm{kB}$ activity in pancreatic CAFs shielded cancer cells from immune attack by increasing CXCL12 expression [59]. Given the wellknown feedforward loop between CXCL12 and STAT3 $[60,61]$, it is possible that STAT3 contributes to NF- $\mathrm{BB}$-mediated immune evasion by this vicious cycle.

\section{STAT3 in immune cells}

STAT3 also plays a pivotal role in a plethora of tumorinfiltrating immune cells that predominantly comprise the TME and recent comprehensive reviews have covered this topic [62-64]. Here we would only like to briefly mention the diverse functions of STAT3 in immune cell milieu, together with some recent advances 
that throw new lights on our understanding of its extremely sophisticated regulation.

Hyperactivation of STAT3 in tumor-infiltrating immune cells causes immunosuppression by inhibiting both innate and adaptive immune responses. In brief, excessive STAT3 activity in innate immune cell subsets may impair the production of pro-inflammatory mediators such as IFN $\gamma$, dampen antigen presentation, and inhibit the tumor-killing activities of effector cells. In adaptive immune subsets, elevated STAT3 activity has the ability to inhibit the accumulation of effector $\mathrm{T}$ cells, thereby restraining their anti-tumor effects [62-64]. Interestingly, some recent studies suggest previously unknown functions of STAT3 in tumor immunity. For instance, placental growth factor (PlGF) [65] and Cxxc finger protein 1 (Cxxc1) [66] can act as key upstream regulators of STAT3 signaling, which subsequently contributes to the differentiation and function of Th17 cells. STAT3 mediates the major impact of $\beta 2$ adrenergic receptor on the immunosuppressive potential of myeloid-derived suppressor cells (MDSCs) in the TME [67]. In glioblastoma-infiltrating tumorassociated macrophages, STAT3 acts as a positive regulator of aryl hydrocarbon receptor (AHR) and thus increases the recruitment of tumor-associated macrophages and tumor growth [68]. It has been shown that STAT3 modulates the abundance and function of regulatory $\mathrm{T}$ (Treg) cells in response to radiation therapy in head and neck cancer, suggesting that STAT3 inhibition may be beneficial for patients receiving radiation [69].

\section{STAT3 in CAFs}

CAFs are the key component of the tumor stroma and contribute to cancer progression and treatment failure by modifying the extracellular matrix, secreting soluble factors, supporting angiogenesis and metastasis, and inhibiting anti-tumor immune responses [70]. There is a growing body of evidence to support that STAT3 can be activated in CAFs by numerous cytokines including leukemia inhibitory factor (LIF) [71]. This STAT3 hyperactivation enables CAFs to produce various immunosuppressive factors such as IL-6, TGF $\beta$, EGF, VEGF, and CCL2, thereby contributing to the pro-oncogenic phenotype of these fibroblasts $[72,73]$. Moreover, a recent study revealed that increased phosphorylation of STAT3 in CAFs is associated with reduced overall survival in colorectal cancer patients, and STAT3 activation in CAFs enhances intestinal tumor growth in vivo [74], exemplifying the importance of STAT3 activation in CAFs for cancer initiation and progression.
STAT3-mediated crosstalk between cancer cells and diverse cell subsets in the TME

Aberrantly activated STAT3 can lead to tumor-induced immunosuppression via propagating the crosstalk between cancer cells and their immunological microenvironment. In tumor cells, hyperactivated STAT3 promotes the expression of immunosuppressive factors such as VEGF, IL6, and IL-10 [53]. Meanwhile, these tumor-derived factors that also happen to be STAT3 activators could be transited to the TME, and thus enhance STAT3 signaling in various immune cell subsets and CAFs (Fig. 2).

In particular, STAT3 hyperactivation in tumor cells has a vital role in dendritic cells (DCs) maturation. DCs essentially are monocytes at a differentiated stage and the key antigen presenting cells of the immune system. As immune sentinels, DCs play an important role in the initiation of $\mathrm{T}$-cell response against tumors, while immature DCs generally induce immune tolerance [75]. Hyperactivated STAT3 in tumor cells can suppress the expression of IL-12 and TNF- $\alpha$, leading to a decrease in Bcl-2 expression in DCs [53]. STAT3 also represses the expression of major histocompatibility complex (MHC) class II complexes and co-stimulatory signals (CD80 and CD86), which are essential to the antigen presenting function of DCs [13]. Meanwhile, STAT3 inhibits DC maturation and innate immunity through negatively regulating the expression of CXCL10 and CCL5 [53]. Furthermore, the immunosuppressive factors such as IL6, IL-10, and VEGF induced by STAT3 can inhibit DC generation through reducing protein kinase $\mathrm{C}$ beta II (PKCBII) expression [76]. Given that immature DCs cannot activate antigen-specific $\mathrm{CD}^{+} \mathrm{T}$ cells, activated STAT3 signaling in tumor cells reduces the antitumorigenic effector functions of $\mathrm{CD} 8^{+} \mathrm{T}$ cells.

What's more, certain factors released by CAFs can modulate STAT3 signaling in other cell types in the tumor milieu. TGF $\beta$, an evolutionarily conserved regulator of tumorigenesis, is a crucial driver of the activity of CAFs. Accumulating evidence suggests that TGF $\beta$ stimulated CAFs increase the secretion of IL- 6 and IL11, which trigger GP130/STAT3 signaling in cancer cells and thus promote cancer metastasis and progression [77-80]. STAT3 is also involved in the crosstalk between CAFs and immune cells. For example, CCL2 secreted from CAFs with STAT3 hyperactivation can promote the recruitment of immunosuppressive MDSCs and hepatocarcinogenesis [72]. Moreover, the differentiation of these recruited MDSCs has been shown to be controlled in an IL-6/STAT3-dependent manner [81]. In addition, IL-6 derived from CAFs can activate STAT3 in DCs, which subsequently induce liver cancer immune escape through impairing $\mathrm{T}$-cell proliferation and promoting Treg cells expansion [82]. STAT3 signaling in CAFs and other cells orchestrates stromal remodeling of 


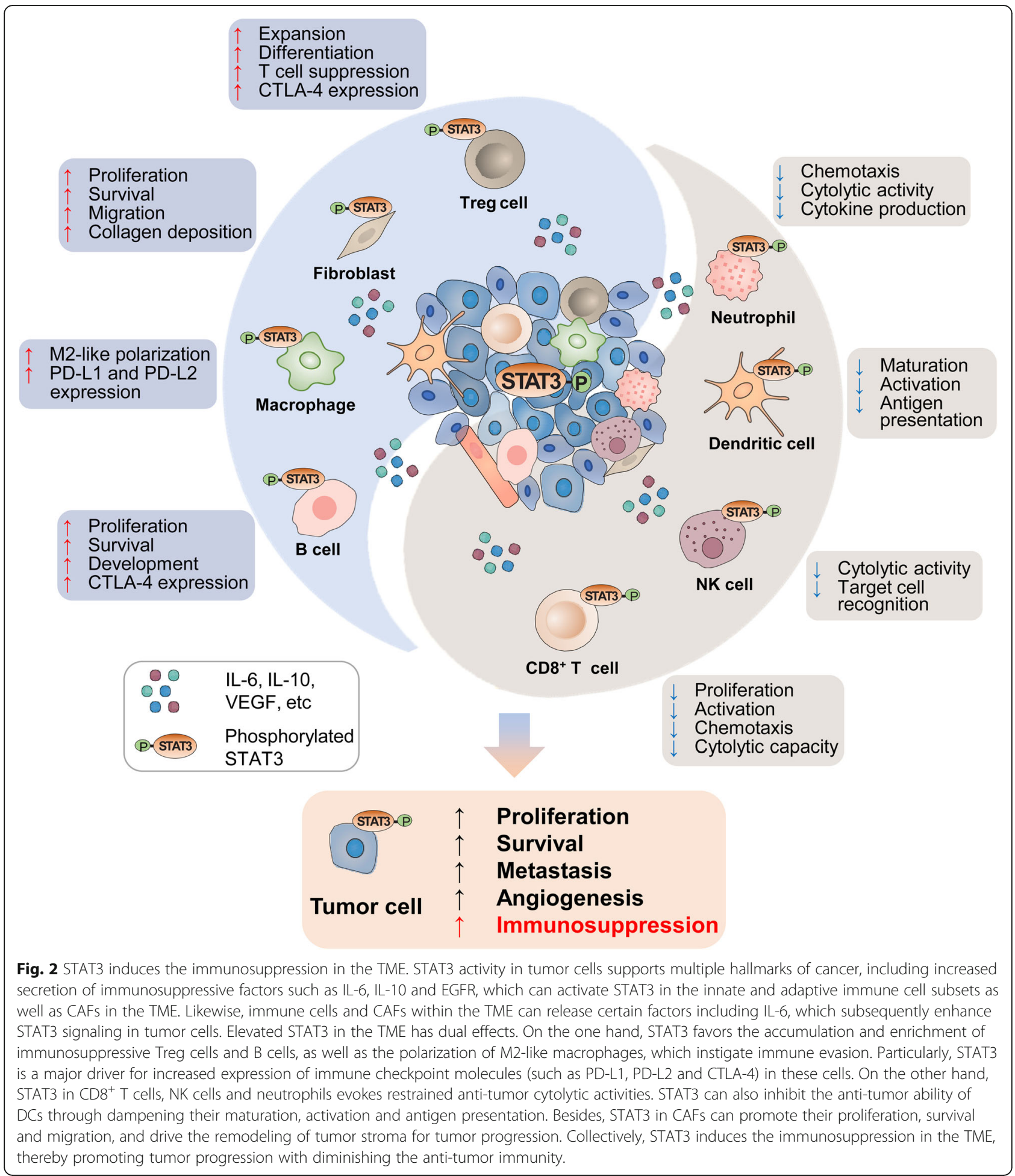

the TME characterized by collagen fibrogenesis, collagen disorganization and fibroblast contractility; the remodeling of the TME is not only important for cancer cell migration and invasion, but also plays a critical role in resistance to therapeutic intervention $[83,84]$.
Overall, the outcome of STAT3-mediated crosstalk between cancer cells and tumor-infiltrating cells within the TME is to promote tumor growth and development, along with diminished anti-tumor immunity (Fig. 2). 
Table 1 Studies of STAT3 inhibitors on pre-clinical cancer models

\begin{tabular}{|c|c|c|c|c|c|c|}
\hline Therapy & Type & Agent & Cell line tested & Mouse model & Functional outcome & Ref \\
\hline \multirow{22}{*}{$\begin{array}{l}\text { Direct } \\
\text { inhibitors }\end{array}$} & \multirow[t]{3}{*}{ Peptides } & DBD-1 & Melanoma, Myeloma & $n / d$ & $\uparrow$ Apoptosis; $\downarrow$ Proliferation & {$[87]$} \\
\hline & & $\begin{array}{l}\text { ISS-610 } \\
\text { prodrugs }\end{array}$ & $B C$ & $n / d$ & $\uparrow$ Apoptosis & [88] \\
\hline & & PY*LKTK & $\mathrm{NIH} 3 \mathrm{~T} 3 / \mathrm{v}-\mathrm{Src}$ or v-Ras & $n / d$ & $\downarrow$ Transformation & [89] \\
\hline & \multirow[t]{17}{*}{ Small molecules } & 60 & $\mathrm{BC}, \mathrm{PC}, \mathrm{PCa}, \mathrm{NSCLC}$ & $n / d$ & $\uparrow$ Apoptosis; $\downarrow$ Proliferation & [90] \\
\hline & & FLLL32 & $B C, P C$ & $\begin{array}{l}\text { Xenograft: MDA-MB-231, } \\
\text { PANC-1 }\end{array}$ & $\begin{array}{l}\uparrow \text { Apoptosis; } \downarrow \text { Proliferation, } \\
\text { Vascularization }\end{array}$ & [91] \\
\hline & & HJC0152 & Glioblastoma & Xenograft: U87 & $\begin{array}{l}\uparrow \text { Apoptosis; } \downarrow \text { Metastasis, } \\
\text { Proliferation }\end{array}$ & [92] \\
\hline & & LL1 & CRC & Xenograft: HCT116 & $\begin{array}{l}\uparrow \text { Apoptosis; } \downarrow \text { Metastasis, } \\
\text { Proliferation }\end{array}$ & [93] \\
\hline & & LLL-3 & BC, Glioblastoma & Xenograft: U87 & $\begin{array}{l}\uparrow \text { Apoptosis; } \downarrow \text { Metastasis, } \\
\text { Proliferation }\end{array}$ & [94] \\
\hline & & LLL12 & $\mathrm{HCC}$ & Xenograft: SNU398 & $\uparrow$ Apoptosis; $\downarrow$ Proliferation & [95] \\
\hline & & LYW-6 & CRC & $\begin{array}{l}\text { AOM/DSS induced CRC } \\
\text { model; Xenograft: HCT116 }\end{array}$ & $\begin{array}{l}\uparrow \text { Apoptosis; } \downarrow \text { Metastasis, } \\
\text { Proliferation }\end{array}$ & [96] \\
\hline & & $\begin{array}{l}\text { Nitidine } \\
\text { chloride }\end{array}$ & Oral cancer & Xenograft: HSC3 & $\uparrow$ Apoptosis; $\downarrow$ Proliferation & [97] \\
\hline & & SD-36 & $\begin{array}{l}\text { BC, CRC, Leukemia, } \\
\text { Lymphoma }\end{array}$ & $\begin{array}{l}\text { Xenograft: MOLM-16, SUP- } \\
\text { M2, SU-DHL-1 }\end{array}$ & $\uparrow$ Apoptosis; $\downarrow$ Proliferation & [98] \\
\hline & & Stattic & $B C$ & $n / d$ & $\uparrow$ Apoptosis & [99] \\
\hline & & STX-0119 & $n / d$ & Humanized NOG-dKO model & $\begin{array}{l}\uparrow \text { Anti-tumor immunity; } \\
\downarrow \text { Proliferation }\end{array}$ & [100] \\
\hline & & S3I-1757 & Melanoma & Xenograft: B16-F10 & $\downarrow$ Proliferation & [101] \\
\hline & & S3I-201 & $B C$ & Xenograft: MDA-MB-231 & $\uparrow$ Apoptosis; $\downarrow$ Proliferation & [102] \\
\hline & & CPA-7 & $\begin{array}{l}\text { BC, CRC, Melanoma, PCa, } \\
\text { NSCLC }\end{array}$ & Xenograft: CT26 & $\uparrow$ Apoptosis; $\downarrow$ Proliferation & [103] \\
\hline & & C48 & BC, CML, Melanoma, PCa & $\begin{array}{l}\text { Xenograft: MDA-MB-468, } \\
\text { C3L5 }\end{array}$ & $\downarrow$ Proliferation & [104] \\
\hline & & GPA512 & PCa & Xenograft: DU145 & $\downarrow$ Proliferation & [105] \\
\hline & & MMPP & $\begin{array}{l}\text { BC, CRC, PCa, HCC, Lung, } \\
\text { Ovary and Skin cancer }\end{array}$ & $\begin{array}{l}\text { Xenograft: Patient-derived } \\
\text { NSCLC, NCI-H460 }\end{array}$ & $\uparrow$ Apoptosis; $\downarrow$ Proliferation & [106] \\
\hline & \multirow[t]{2}{*}{ Oligonucleotides } & $\begin{array}{l}\text { InS3- } \\
54 \mathrm{~A} 18\end{array}$ & $\mathrm{BC}, \mathrm{NSCLC}$ & Xenograft: A549 & $\downarrow$ Metastasis, Proliferation & [107] \\
\hline & & $\begin{array}{l}\text { STAT3 } \\
\text { hpdODN }\end{array}$ & CRC & $n / d$ & $\downarrow$ Proliferation & [108] \\
\hline \multirow{4}{*}{$\begin{array}{l}\text { Indirect } \\
\text { inhibitors }\end{array}$} & \multirow[t]{2}{*}{ JAK2 } & INCB16562 & Leukemia & MPLW515L model & $\downarrow$ Proliferation & [109] \\
\hline & & TG101209 & Leukemia & AML1-ETO9a leukemia model & $\uparrow$ Apoptosis; $\downarrow$ Proliferation & [110] \\
\hline & EGFR & JND3229 & $\mathrm{BaF} 3$ & Xenograft: BaF3-EGFR & $\downarrow$ Proliferation & [111] \\
\hline & FGFR, VEGFR & ODM-203 & Bladder cancer, NSCLC, GC & $\begin{array}{l}\text { Xenograft: H1581, KMS11, } \\
\text { RT4, SNU16 }\end{array}$ & $\begin{array}{l}\uparrow \text { Anti-tumor immunity; } \\
\downarrow \text { Metastasis, Proliferation }\end{array}$ & [112] \\
\hline \multirow[t]{5}{*}{ Combination } & Direct inhibitor & HJC0152 & \multirow[t]{2}{*}{$\mathrm{BC}, \mathrm{THP} 1$} & \multirow[t]{2}{*}{ Xenograft: 4T1 } & \multirow{2}{*}{$\begin{array}{l}\uparrow \text { Anti-tumor immunity; } \\
\downarrow \text { Proliferation }\end{array}$} & \multirow[t]{2}{*}{ [113] } \\
\hline & STING agonist & $\begin{array}{l}\text { c-diAM } \\
(P S) 2\end{array}$ & & & & \\
\hline & JAK1/2 inhibitor & Ruxolitinib & \multirow[t]{2}{*}{ PC } & \multirow[t]{2}{*}{ Xenograft: PANCO2-H7 } & \multirow{2}{*}{$\begin{array}{l}\uparrow \text { Anti-tumor immunity; } \\
\downarrow \text { Proliferation }\end{array}$} & \multirow[t]{2}{*}{ [114] } \\
\hline & $\begin{array}{l}\text { Anti-PD-1 } \\
\text { antibody }\end{array}$ & RMP1-14 & & & & \\
\hline & $\begin{array}{l}\text { SRC, } A B L \\
\text { inhibitor }\end{array}$ & Dasatinib & $n / d$ & Tgfbr1/Pten 2cKO model & $\begin{array}{l}\uparrow \text { Anti-tumor immunity; } \\
\downarrow \text { Proliferation }\end{array}$ & [115] \\
\hline
\end{tabular}


Table 1 Studies of STAT3 inhibitors on pre-clinical cancer models (Continued)

\begin{tabular}{llllll}
\hline Therapy & Type & Agent & Cell line tested & Mouse model & Functional outcome \\
\hline $\begin{array}{l}\text { VEGFR2 } \\
\text { antibody }\end{array}$ & DC101 & $\mathrm{n} / \mathrm{d}$ & Xenograft: LLC, CT26 & $\begin{array}{l}\downarrow \text { Proliferation; } \uparrow \text { Anti-tumor } \\
\text { immunity, Vascular normalization }\end{array}$ \\
& & & \\
STING agonist & $\begin{array}{l}\text { CGAMP, } \\
\text { RR-CDA }\end{array}$ & & & \\
\hline
\end{tabular}

AOM/DSS Azoxymethane/dextran sodium sulfate, BC Breast cancer, CML Chronic myelogenous leukemia, CRC Colorectal cancer, GC Gastric cancer, HCC Hepatocellular carcinoma, LLC Lewis lung carcinoma, MPLW515L Somatic mutations at codon 515 of the thrombopoietin receptor, NSCLC Non-small cell lung cancer, $P C$ Pancreatic cancer, $P C a$ Prostate cancer, $n / d$ Not determined, hpdODN hairpin decoy oligodeoxynucleotide

\section{Targeting STAT3 for cancer immunotherapy} Direct targeting of STAT3

Although targeting STAT3 has been extensively investigated for decades, this field still remains largely unexplored. The most common approach in targeting STAT3 directly is to prevent the formation of functional STAT3 dimers through disrupting the domains of $\mathrm{SH} 2, \mathrm{DBD}$, or NTD $[85,86]$. In general, direct inhibitors of STAT3 can be classified into three categories: peptides, small molecules and oligonucleotides. Studies of these inhibitors on pre-clinical cancer models are summarized in Table 1 [87-112] and relevant ongoing clinical trials are introduced in Table 2 [117-119].

Peptides are usually designed based on the structure of amino acid residues in STAT3 protein and can be directed towards different domains. Phosphopeptide inhibitor (PY*LKTK), derived from the binding peptide sequence of the STAT3-SH2 domain, represents the first successful attempt to disrupt STAT3 dimerization [89]. However, the further development of peptide for the clinical use is currently limited due to their poor cellular permeability and lack of stability in vivo, and even the second-generation peptidomimetics are largely suffering from similar limitations [127].

Non-peptide small molecules capable of disrupting phosphorylation of STAT3 or STAT3-STAT3 dimerization have recently emerged as an attractive alternative approach to the above. These small molecule inhibitors usually selectively bind to the SH2, the DBD, or the NTD domain of STAT3 to block transcription of target genes [85]. BBI608 (Napabucasin), a small molecule inhibitor that selectively binds to the DBD domain of STAT3, is the only direct STAT3 inhibitor that has advanced into phase III trials thus far. The excellent outcome of a recent phase III monotherapy trial suggested that BBI608 has potential implication in advanced colorectal cancer [117]. Moreover, FDA has approved BBI608 as an orphan drug for treatment of gastric and pancreatic cancer based on the promising results in phase I/II clinical trials.

Numerous small molecule inhibitors of STAT3 have been identified by virtual screening. Of note, although these inhibitors exhibit excellent physicochemical properties in vitro, most of them show poor clinical efficacy, which might be due to low aqueous solubility and low cell permeability [86]. Several novel approaches have recently emerged to overcome this dilemma, and show great promise to yield therapeutic agents to targeting transcription factors, including STAT3. For instance, the small-molecule proteolysis-targeting chimera (PROTAC)-based strategy has attracted a lot of attention because it can inhibit target protein function as well as counteract increased target protein expression [128]. The studies on PROTAC-mediated degradation of oncogenic proteins such as BRD4 [129], BCR-ABL [130], receptor tyrosine kinase (RTK) [131], and BCL$X_{L}$ [132] have shown encouraging results, suggesting the potential clinical applicability of this ingenious approach. SD-36, a novel inhibitor identified by the PROTAC-based strategy, exhibits high selectivity for STAT3 and high cell permeability [98]. Moreover, SD-36 treatment can cause a profound and longlasting suppression of tumor in mouse models of leukemia and lymphoma [98], suggesting that PROTAC-based strategy may be a promising and reliable avenue for searching small molecule inhibitors against STAT3. Further, the outstanding performance of SD-36 in cancer treatment suggests that the strategy of targeting STAT3 protein degradation may be superior to suppress STAT3 expression.

Oligonucleotides represent a new treatment strategy for 'undruggable' cancer targets such as STAT3. STAT3-binding decoy oligodeoxynucleotides, can sequester STAT3 and thus decrease its binding to cognate DNA sites within target genes [133]. Antisense oligonucleotides (ASOs) are designed to block STAT3 activity by targeting STAT3 mRNA. For example, AZD9150, a second-generation STAT3 ASO, targets the 3'-untranslated region (3'-UTR) of the STAT3 gene [134]. Preclinical testing and clinical evaluation have revealed the high efficacy and low toxicity of AZD9150 in oncotherapy $[135,136]$. Although oligodeoxynucleotides inhibitors of STAT3 provide exquisite specificity and potency, their poor cell membrane penetrance, rapid degradation, and the lack of effective targeted delivery carriers, remain the major obstacles that impede their use in solid tumors. Aptamers have also emerged as useful targeted delivery agents for conventional drugs and small RNAs including siRNAs and miRNAs due to several advantages, such as small physical size, high stability and low immunogenicity [137]. 
Table 2 STAT3 inhibitors in currently on-going clinical trials

\begin{tabular}{|c|c|c|c|c|c|c|}
\hline Therapy & Type & Agent & Indication & Phase & NCT number & Ref \\
\hline \multirow{14}{*}{$\begin{array}{l}\text { Direct } \\
\text { inhibitors }\end{array}$} & \multirow[t]{12}{*}{ Small molecules } & \multirow[t]{2}{*}{ BBI608 (FDA approved) } & Advanced malignancies & $|/| \mid$ & NCT01775423 & NA \\
\hline & & & CRC & III & NCT01830621 & {$[117]$} \\
\hline & & Celecoxib* (FDA approved) & CRC & III & NCT00087256 & NA \\
\hline & & C188-9 & $\begin{array}{l}\text { BC, CRC, HNSCC, HCC, NSCLC, GAC, Melanoma, } \\
\text { Advanced cancer }\end{array}$ & । & NCT03195699 & NA \\
\hline & & \multirow[t]{2}{*}{ OPB-111077 } & Acute myeloid leukemia & । & NCT03197714 & NA \\
\hline & & & Advanced HCC & । & NCT01942083 & NA \\
\hline & & \multirow[t]{2}{*}{ OPB-31121 } & Advanced cancer, Solid tumors & 1 & NCT00955812 & NA \\
\hline & & & $\mathrm{HCC}$ & $|/| \mid$ & NCT01406574 & NA \\
\hline & & \multirow[t]{3}{*}{ OPB-51602 } & Malignant solid tumors & । & NCT01184807 & NA \\
\hline & & & Hematological malignancies & 1 & NCT01344876 & NA \\
\hline & & & Nasopharyngeal carcinoma & । & NCT02058017 & NA \\
\hline & & $\begin{array}{l}\text { Pyrimethamine* (FDA } \\
\text { approved) }\end{array}$ & CLL, Small lymphocytic lymphoma & $|/| \mid$ & NCT01066663 & NA \\
\hline & \multirow[t]{2}{*}{ Oligonucleotides } & AZD9150 & Lymphoma & $|/| \mid$ & NCT01563302 & [118] \\
\hline & & STAT3 decoy & Head and neck cancer & 0 & NCT00696176 & [119] \\
\hline \multirow{21}{*}{$\begin{array}{l}\text { Indirect } \\
\text { inhibitors }\end{array}$} & \multirow[t]{4}{*}{ JAK1/2 } & AZD-1480 & Solid tumors & 1 & NCT01112397 & NA \\
\hline & & \multirow[t]{2}{*}{ CYT 387} & Myelofibrosis & $|/| \mid$ & NCT01423058 & [120] \\
\hline & & & PMF, Post-PV, Post-ET MF & III & NCT02101268 & NA \\
\hline & & Ruxolitinib (FDA approved) & Myelofibrosis & $\|$ & NCT03427866 & NA \\
\hline & \multirow[t]{2}{*}{ JAK2 } & LY2784544 & Myeloproliferative neoplasms & $\|$ & NCT01594723 & {$[121]$} \\
\hline & & SB1518 & Myelofibrosis & III & NCT02055781 & {$[122]$} \\
\hline & \multirow[t]{3}{*}{ EGFR } & Cetuximab (FDA approved) & Metastatic CRC & $|/| \mid$ & NCT02117466 & NA \\
\hline & & \multirow{2}{*}{$\begin{array}{l}\text { Panitumumab (FDA } \\
\text { approved) }\end{array}$} & Advanced CRC & $\|$ & NCT03311750 & NA \\
\hline & & & Metastatic CRC & IV & NCT02301962 & NA \\
\hline & \multirow[t]{2}{*}{ FGFR } & \multirow[t]{2}{*}{ Ponatinib (FDA approved) } & CML & $\|$ & NCT04043676 & NA \\
\hline & & & $C M L, A L L$ & $\|$ & NCT04233346 & NA \\
\hline & \multirow[t]{2}{*}{ IL-6R } & Siltuximab (FDA approved) & Multiple myeloma & $\|$ & NCT03315026 & NA \\
\hline & & Tocilizumab (FDA approved) & $\mathrm{HCC}$ & $|/| \mid$ & NCT02997956 & NA \\
\hline & VEGF & Bevacizumab (FDA approved) & Metastatic CRC & $\|$ & NCT02226289 & NA \\
\hline & VEGFR & Apatinib & Lung carcinoma & $\|$ & NCT03709953 & NA \\
\hline & VEGFR, PDGFR & Sorafenib (FDA approved) & Advanced HCC & IV & NCT02733809 & NA \\
\hline & \multirow[t]{2}{*}{ VEGFR, PDGFR, c-KIT } & \multirow[t]{2}{*}{ Sunitinib (FDA approved) } & Clear cell renal carcinoma & $\|$ & NCT03066427 & NA \\
\hline & & & Pancreatic neuroendocrine tumor metastatic & $\|$ & NCT02713763 & NA \\
\hline & $\mathrm{SRC}, \mathrm{ABL}$ & Dasatinib (FDA approved) & Chronic-phase CML & IV & NCT01660906 & [123] \\
\hline & \multirow[t]{2}{*}{ SRC } & Bosutinib (FDA approved) & CML & $\|$ & NCT02810990 & NA \\
\hline & & KX2-391 & Bone-metastatic, Castration-resistant PCa & $\|$ & NCT01074138 & {$[124]$} \\
\hline \multirow[t]{6}{*}{ Combination } & \multirow{6}{*}{$\begin{array}{l}\text { Direct inhibitors and } \\
\text { ICB }\end{array}$} & \multirow{4}{*}{$\begin{array}{l}\text { AZD9150, Durvalumab (anti- } \\
\text { PD-L1) }\end{array}$} & NSCLC & $\|$ & NCT03334617 & NA \\
\hline & & & PC, CRC, NSCLC & $\|$ & NCT02983578 & NA \\
\hline & & & Advanced solid tumors, Metastatic HNSCC & $|/| \mid$ & NCT02499328 & NA \\
\hline & & & Diffuse large B-cell lymphoma & I & NCT02549651 & NA \\
\hline & & $\begin{array}{l}\text { BBl608, Nivolumab (anti-PD- } \\
\text { L1) }\end{array}$ & Metastatic CRC & $\|$ & NCT03647839 & NA \\
\hline & & BBI608, Pembrolizumab (anti- & Metastatic CRC & $|/| \mid$ & NCT02851004 & NA \\
\hline
\end{tabular}


Table 2 STAT3 inhibitors in currently on-going clinical trials (Continued)

\begin{tabular}{|c|c|c|c|c|c|c|}
\hline Therapy & Type & Agent & Indication & Phase & NCT number & Ref \\
\hline & \multirow{9}{*}{$\begin{array}{l}\text { Indirect inhibitors } \\
\text { and ICB }\end{array}$} & Apatinib, SHR-1210 (anti-PD-1) & Melanoma & $\|$ & NCT03955354 & NA \\
\hline & & $\begin{array}{l}\text { Bevacizumab, Atezolizumab } \\
\text { (anti-PD-L1) }\end{array}$ & Unresectable HCC & III & NCT03434379 & [125] \\
\hline & & $\begin{array}{l}\text { Cetuximab, Pembrolizumab } \\
\text { (anti-PD-1) }\end{array}$ & Recurrent or metastatic HNSCC & $\|$ & NCT03082534 & NA \\
\hline & & $\begin{array}{l}\text { Dasatinib, Ipilimumab (anti- } \\
\text { CTLA-4) }\end{array}$ & GIST, Stage III /IV soft tissue sarcoma & । & NCT01643278 & [126] \\
\hline & & $\begin{array}{l}\text { Dasatinib, Nivolumab (anti- } \\
\text { PD-L1) }\end{array}$ & Philadelphia chromosome positive ALL & । & NCT02819804 & NA \\
\hline & & \multirow{2}{*}{$\begin{array}{l}\text { Ruxolitinib, Pembrolizumab } \\
\text { (anti-PD-1) }\end{array}$} & Hematological malignancies & $\|$ & NCT04016116 & NA \\
\hline & & & Metastatic stage IV TNBC & I & NCT03012230 & NA \\
\hline & & $\begin{array}{l}\text { Sorafenib, BGB-A317(anti-PD- } \\
\text { 1) }\end{array}$ & $\mathrm{HCC}$ & III & NCT03412773 & NA \\
\hline & & $\begin{array}{l}\text { Sorafenib, Nivolumab (anti- } \\
\text { PD-L1) }\end{array}$ & Advanced or metastatic HCC & $\|$ & NCT03439891 & NA \\
\hline & $\begin{array}{l}\text { Indirect inhibitor and } \\
\text { CAR-T }\end{array}$ & Tocilizumab, CAR-T 19 & Lymphoblastic leukemia & NA & NCT02906371 & NA \\
\hline
\end{tabular}

ALL Acute lymphoblastic leukemia, BC Breast cancer, Celecoxib* An FDA approved nonsteroidal anti-inflammatory drug, CML Chronic myelogenous leukemia, CLL Chronic lymphocytic leukemia, CRC Colorectal cancer, HNSCC Head and neck squamous cell carcinoma, NA Not available, NSCLC Non-small cell lung cancer, HCC Hepatocellular carcinoma, GAC Gastric adenocarcinoma, Pyrimethamine* An FDA approved anti-parasitic drug, PMF Primary myelofibrosis, Post-PV Postpolycythemia vera, Post-ET MF Post-essential thrombocythemia myelofibrosis, PC Pancreatic cancer, PCa Prostate cancer, GIST Gastrointestinal stromal tumor, TNBC Triple negative breast cancer

Recently, STAT3 silencing by aptamer-siRNA chimera obtained excellent inhibition in the therapy of glioblastoma [138, 139], suggesting that the improved oligonucleotides might offer translational potential for the treatment of solid tumors.

Since STAT3 is a transcription factor, it is traditionally regarded as an undruggable target. Direct targeting of STAT3 has proven to be considerably challenging, owing in part to high sequence similarity with the other STAT members [86, 140]. Moreover, several issues such as high toxicity and poor bioavailability have become significant impediments to the clinical development of direct STAT3 inhibitors [86]. Interestingly, some FDAapproved compounds, such as Pyrimethamine and Celecoxib, have been identified as STAT3 inhibitors through drug-repositioning screening [141, 142]. These findings not only provide another source for searching STAT3 inhibitors, but also suggest potential applications of these drugs in cancer therapy. In addition, similar to combined therapy, certain bifunctional compounds are emerging and may represent a new generation of highly efficacious STAT3 inhibitors for cancer therapy in the future. For example, the compound $8 \mathrm{u}$ has dual immunotherapeutic and anticancer efficacy through simultaneously inhibiting indoleamine-2,3-dioxygenase 1 (IDO1) and STAT3 [143].

\section{Indirect targeting of STAT3}

In parallel with direct inhibitors, indirect inhibitors of STAT3 have been pursued by targeting the upstream or downstream components of the STAT3 signaling pathway, and hundreds of leading compounds have been identified [144-148]. Out of those, Ruxolitinib, Dasatinib and Siltuximab that target JAK, SRC/ABL, and IL-6 respectively, have been approved by FDA for cancer therapy. Indirect STAT3 inhibitors in currently on-going clinical trials are summarized in Table 2 [120-124]. Of note, indirect STAT3 inhibitors lack specificity for STAT3 and may cause fairly extensive kinase inhibition because the targeted molecules are often involved in intricate signaling pathways.

Intriguingly, it has recently been shown that phosphorylated STAT3 is present in exosomes from 5-fluorouracil (5-FU) resistant colorectal cancer cells, which contributes to acquired 5-FU resistance [149]. Given the importance and efficiency of exosomes in intercellular and interorgan communication $[150,151]$, these findings not only add another complexity to STAT3 regulation, but also pave a new way to inhibit the oncogenic function of STAT3, as well as to delivery STAT3 inhibitors via exosomes.

\section{Integrating STAT3 in combination cancer immunotherapy}

Immunotherapy is currently among the most promising approaches for cancer treatment. This therapeutic strategy, represented mainly by immune checkpoint blockade (ICB) and chimeric antigen receptor $\mathrm{T}$ cells (CAR-T), has obtained unprecedented results in patients with previously incurable cancers $[3,152]$. However, there are some key challenges that need to be resolved urgently, 
a

STAT3 direct inhibitors
Peptides
Small molecules
Oligonucleotides

b

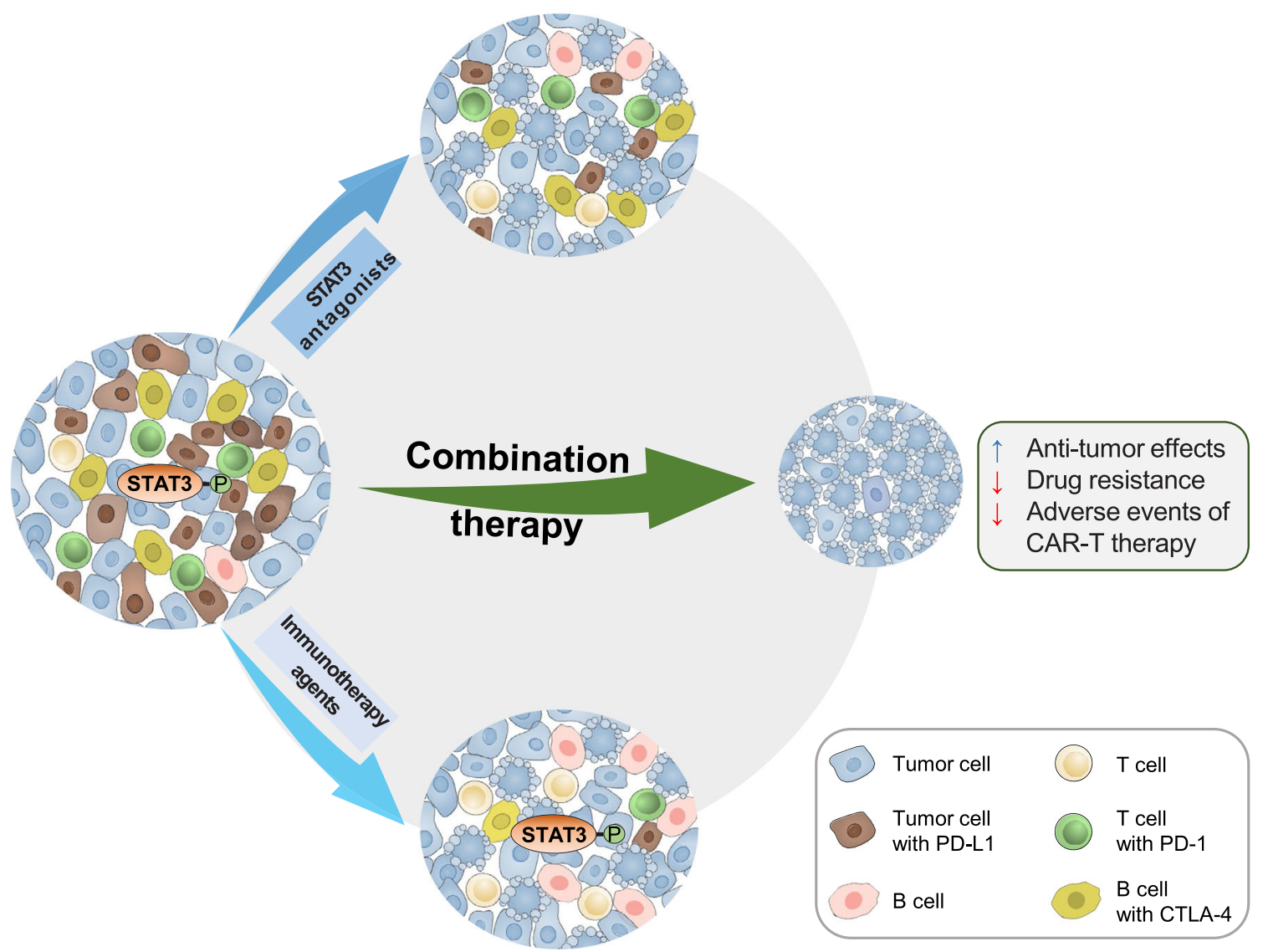

Fig. 3 (See legend on next page.) 


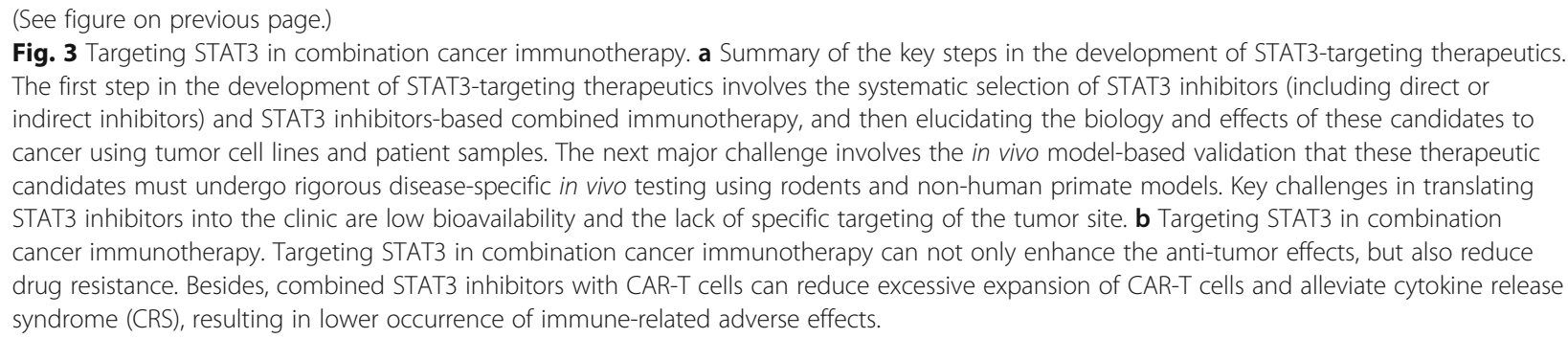

including limited clinical response rates and significant autoimmune-related side effects [3, 153]. For instance, ICB has shown remarkable effectiveness in solid tumors including melanoma, non-small cell lung cancers and renal cancer, however, even in these cancers, the majority of patients still do not respond to the treatment [3]. Furthermore, certain types of cancer such as pancreatic cancer and prostate cancer show resistance to immune checkpoint inhibition therapy [3, 154]. Thus, combination therapy is considered to be a promising direction for improving outcomes for cancer treatment. Preclinical and clinical data suggest that combination cancer immunotherapies have enhanced therapeutic efficacy and reduced drug resistance compared with monotherapy $[155,156]$. These encouraging data has triggered many investigations of combination strategies, and the combination of STAT3 inhibitors with other immunotherapy agents are also emerging (Fig. 3).

\section{Combined blockade of STAT3 and immune checkpoint}

Up-regulated expression of the immune checkpoint molecules, including CTLA-4, PD-1, and PD-L1, has been documented to facilitate tumor immune escape. A substantial amount of evidence has shown that STAT3 is able to directly or indirectly regulate these immune checkpoint molecules. As a transcription factor, STAT3 can increase expression of PD-1, PD-L1, and PD-L2 by direct binding to their promoters [157-160]. Meanwhile, STAT3 has been identified to indirectly induce expression of immune checkpoint molecules through modulating diverse signaling pathways. For example, STAT3 increased CTLA-4 expression in tumor-associated B cells in a JAK-dependent manner [161] and enhanced CTLA-4 expression in Treg cells through IL-10 [162]. In addition, STAT3 mediated HDAC6-induced PD-L1 expression in osteosarcoma cells [163]. Conversely, recent evidence also suggests a role of immune checkpoint molecules in STAT3 expression. Celada et al. reported that PD-1 upregulation in $\mathrm{CD}^{+}{ }^{+} \mathrm{T}$ cells leads to an increase in STAT3 mRNA expression by undescribed mechanism, and the latter is required for IL-17 and TGF $\beta 1$ production [164]. Interestingly, an early study from the same research group showed PD-1 can attenuate TCR-dependent activation of PI3K/AKT pathway in
$\mathrm{CD} 4^{+} \mathrm{T}$ cells [165]. Given PI3K/AKT as a known repressor of STAT3 transcription [166], it is likely that PD-1 indirectly enhances STAT3 expression through inhibition of PI3K. The reciprocal regulation of STAT3 and immune checkpoint molecules not only suggests an involvement of STAT3 in anti-tumor immunity, but also provides a promising strategy to improve the efficacy of current immune checkpoint inhibitors.

Combined blockade of STAT3 and immune checkpoint has shown encouraging results, whereby the addition of STAT3 inhibitors can enhance therapeutic efficacy, and reduce resistance to ICB immunotherapy in parallel. Dasatinib, an indirect STAT3 inhibitor against SRC/ABL, significantly facilitated anti-CTLA-4 immunotherapy in head and neck squamous cell carcinoma [115], while the combined blockade of IL-6 and PD-L1 remarkably inhibited the growth of pancreatic ductal adenocarcinoma and hepatocellular carcinoma (HCC) $[167,168]$. The resistance to anti-PD-1 antibodies can be overcome by treatment with JAK inhibitor in mice with pancreatic orthotopic tumors [114]. Niclosamide blocked STAT3-induced PD-L1 transcription, and thus enhanced the efficacy of anti-PD-1/PD-L1 antibodies in non-small cell lung cancer [169]. More recently, a phase III trial reported exciting results of STAT3-based combination therapy in treatment of advanced HCC. Compared to the first-line drug sorafenib, the combination of bevacizumab (a monoclonal antibody targeting VEGF) and atezolizumab (a PD-L1 inhibitor) can significantly prolong the overall survival and progression-free survival of patients with unresectable HCC, along with comparable adverse effects [125]. In addition, certain STAT3 inhibitors, such as BBI608 and AZD9150, combining with immune checkpoint inhibitors, are currently being tested in pre-clinical (Table 1) and clinical trials (Table 2) [126]. The promising early phase clinical trials encourage further clinical development of this combination strategy.

\section{Combined STAT3 inhibitors and CAR-T}

CAR-T cell therapy, a rapidly emerging and effective immunotherapeutic approach, has revolutionized anti-cancer therapies for hematologic malignancies, especially acute lymphoblastic leukemia and lymphoma [152]. Two Anti- 
CD19 CAR-T therapies are currently approved by FDA for the treatment of CD19-positive leukemia or lymphoma. Although the efficacy of CAR-T therapy in solid tumors has lagged far behind, a great number of CAR-T trials are ongoing and positive outcomes are increasingly being reported for multiple solid tumors, including glioblastoma, gastrointestinal, genitourinary, breast, and lung cancer [170]. For example, CAR-T cells targeting B7-H3, a transmembrane protein belonging to the $\mathrm{B} 7 \mathrm{immune}$ family, inhibited the growth of neuroblastoma, pancreatic and ovarian cancer in vitro and in xenograft mouse models without evident toxicity $[171,172]$.

The involvement of STAT3 signaling in CAR-T therapy is emerging. Transcriptomic profiling showed that anti-CD19 CAR-T cells from responsive patients with chronic lymphocytic leukemia had an increased IL-6/ STAT3 signature, which promoted the expansion of CAR-T cells [173]. In line with this, a novel anti-CD19 CAR-T cells with constitutive activation of STAT3 showed increased proliferation and reduced terminal differentiation of CAR-T cells, and conferred superior antitumor effects [174]. Similarly, CAR-T cells expressing the ectodomain of the IL-4 receptor and the end domain of the IL-21 receptor activated the STAT3 pathway and enhanced Th17-like polarization, representing a potential clinical CAR-T therapy for solid tumors enriching IL-4 [175]. These studies suggest a beneficial effect of STAT3 activation in CAR-T cells.

As mentioned above, STAT3 hyperactivation in tumor stroma is immunosuppressive and can increase the expression of certain cytokines and growth factors. Accordingly, constitutive expression of an array of cytokines such as IL-6 and IL-10 potentially could increase the risk of serious adverse events of CAR-T therapy including cytokine release syndrome [176]. Thus, there are some attempts to combine CAR-T therapy with STAT3 inhibitors for improving the persistence and anti-tumor effects, as well as negating toxicities of CRA-T cells in vivo. For instance, the JAK2/STAT3 axis is a crucial driver of liver-associated MDSCs and inhibition of STAT3 increased the efficacy of CAR-T cells in liver cancer metastasis [177]. In addition, a clinical study is currently on-going, which tests the efficacy and administration of the anti-IL-6 therapy (tocilizumab) on anti-CD19 CAR-T cells associated cytokine release syndrome (NCT02906371).

\section{Combined STAT3 inhibitors and STING agonists}

Stimulator of interferon genes (STING) is a major adaptor protein that plays an important role in anti-viral and anti-tumor immunity [178]. When stimulated by cytosolic DNA, STING activates TANK-binding kinase 1 (TBK1), which subsequently phosphorylates interferon regulatory factor 3 (IRF3) to promote IFN expression
[178]. Activated STING can propagate interferon receptor signaling in tumor-infiltrating DCs and elicit $\mathrm{CD} 8^{+} \mathrm{T}$ cells against tumor-associated antigens in vivo [179]. Therefore, STING agonists are of continuing research interest as novel adjuvants to boost cancer immunotherapy. A recent study showed that STING-activating nanoparticles (STING-NPs) can convert immunosuppressive tumors to immunogenic microenvironments and then induce anti-tumor immune responses and immunological memory in mice with melanoma [180]. In another study, Ramanjulu et al. reported that STING agonist can lead to complete and lasting regression of tumors in mice with colon tumors [179]. These encouraging results of preclinical studies point towards the potential for improving clinical outcomes of immunotherapy, and some STING agonists such as cdiAM (PS) 2 and cGAMP are currently being evaluated in clinical trials (NCT03937141, NCT02986867).

Several previous studies have suggested potential interactions between STING signaling and STAT3-driven oncogenic pathways [181-183]. It has been observed that rapid colorectal cancer progression in STINGdeficient mice is associated with STAT3 hyperactivation [181]. Further research found that STING plays a vital role in regulation of MDSC differentiation and antitumor immunity in nasopharyngeal carcinoma by increasing the expression of SOCS1, a classic repressor of STAT3 [182]. Besides, TBK1, which is activated by cytosolic DNA in a STING-dependent manner, can restrain activation of STAT3 through direct phosphorylation of STAT3 at serine 754 in the TAD [183].

Recent evidence indicates that the combination of STING agonists with STAT3 inhibitors can enhance tumor immunogenicity and optimize the immunotherapeutic effects $[113,116]$. For example, combined STAT3 direct inhibitor HJC0152 with STING agonist c-diAM (PS)2 increased CD8 ${ }^{+}$ $\mathrm{T}$ cells, reduced Treg cells and MDSCs in the TME, and thus effectively enhanced anti-tumor immunity in mice with breast cancer [113]. A preclinical study demonstrated the combination of STING agonist (cGAMP or RR-CDA) with the indirect STAT3 inhibitor VEGFR2 was maximally effective for immunotherapy-resistant tumors in breast and lung cancer [116].

STAT3 blockade can also markedly improve other effective immunotherapeutic approaches including cancer vaccines and immunostimulatory Toll-like receptor (TLR) agonists (such as CpG oligodeoxynucleotides). For example, a novel strategy combining STAT3 ASO with TLR9 stimulation (CpG oligonucleotide) has been shown to enhance the anti-tumor immunity and overcome tumor immune tolerance in prostate cancer [184]. The combination of STAT3 inhibitor and DC-based vaccine led to improved therapeutic outcomes in mouse colon cancer [185]. The beneficial outcomes of these 
immunotherapy combinations about STAT3 inhibitors warrant further clinical validation. Notably, STAT3 antagonists, either direct or indirect STAT3 inhibitors, are generally less likely to completely block STAT3 signaling and might not trigger severe autoimmune disorders. However, it cannot be ignored that the use of STAT3 inhibitors and other immunotherapy agents in combination may result in more frequent and severe immunerelated adverse events (irAEs) compared to monotherapy. Accordingly, risk evaluations for irAEs should be part of the decision criteria for determining immunotherapy combinations. Likewise, early recognition and adequate management for irAEs are indispensable to minimize treatment-related serious complications.

\section{Conclusions and perspectives}

STAT3 becomes excessively activated in multiple human cancers, and acts as a crucial signaling node for tumor cells and TME comprising cells, especially tumorinfiltrating immune cells. Therefore, targeting STAT3 is expected to offer multiple benefits, including reduced tumor cell intrinsic proliferation, enhanced anti-tumor effects of tumor-infiltrating immune cells, and improved the immunosuppressive crosstalk within the TME. These effects have positioned STAT3 as an arisen potential promising target for cancer therapy.

To date, many endeavors have been made to target STAT3 for the development and application of new drugs. These approaches are devised to inhibit STAT3 directly by peptides, small molecules and decoy oligonucleotides, or indirectly by blocking upstream signaling pathways such as IL-6 and JAK2 pathways. Currently, the core idea of direct targeting STAT3 is to prevent the formation of functional STAT3 dimers through disrupting phosphorylation of STAT3. Beyond phosphorylation, other posttranslational modifications, such as acetylation, methylation and sumoylation, are emerging to modulate STAT3 activation through diverse mechanisms, providing a broadened list of candidate regulatory targets for the STAT3 inhibitors.

To improve the response rate and the number of responding cancer types, combined immunotherapies are now being undertaken. Combination therapies of STAT3 inhibitors with currently therapeutic anti-tumor drugs including the immune-checkpoint inhibitors may open up new possibilities for long-lasting and multilayered tumor control. Although preclinical studies and early clinical trials on combined blockade of STAT3 and immune checkpoint have shown encouraging results, their clinical outcomes await further investigation. Moreover, predictive biomarkers are urgently required to rationally incorporate STAT3 inhibitors into the combination immunotherapy. The ncRNAs, particularly miRNAs, might prove to be potentially promising predictive biomarkers that can provide a basis for improved precision medicine, though the related studies are currently not explored in depth.

In summary, therapeutically targeting mediators of the STAT3 signaling, which has already been shown to be beneficial in the restoration of anti-tumor immunity, provides attractive avenues that are currently being explored for the immunotherapy of cancers both as monotherapy and in combination therapies.

\section{Abbreviations}

AHR: Aryl hydrocarbon receptor; ALL: Acute lymphoblastic leukemia; AOM: Azoxymethane; ASOs: Antisense oligonucleotides; BC: Breast cancer; BCl-2: B-cell lymphoma-2; BRD4: Bromodomain-containing protein 4; CAFs: Cancer-associated fibroblasts; CAR-T: Chimeric antigen receptor T cells; CCD: Coiled-coil domain; CCL: C-C motif ligand; CD80/86: Cluster of differentiation 80/86; circRNAs: Circular RNAs; CLL: Chronic lymphocytic leukemia; CML: Chronic myelogenous leukemia; CRS: Cytokine release syndrome; CTLA-4: Cytotoxic T-lymphocyte-associated protein 4; CXCL: C-X-C motif chemokine ligand; Cxxc1: Cxxc finger protein 1; DBD: DNA-binding domain; DCs: Dendritic cells; DSS: Dextran sodium sulfate; DUSP22: Dual specificity phosphatase 22; EGF: Epidermal growth factor; EGFR: Epidermal growth factor receptor; EZH2: Enhancer of zeste homolog 2; FGFR: Fibroblast growth factor receptor; GAC: Gastric adenocarcinoma; GC: Gastric cancer; GIST: Gastrointestinal stromal tumor; GP130: Glycoprotein 130; HADC: Histone deacetylase; HCC: Hepatocellular carcinoma; HIF1a: Hypoxia-inducible factor 1a; HNSCC: Head and neck squamous cell carcinoma; hpdODN: Hairpin decoy oligodeoxynucleotide; ICB: Immune checkpoint blockade; IDO1: Indoleamine-2,3-dioxygenase 1; IFNs: Interferons; IFN-Is: Type I interferons; IL: Interleukin; LoxI3: Lysyl oxidase-like 3; irAEs: Immune-related adverse events; IRF3: Interferon regulatory factor 3; ISGF3: Interferonstimulated gene factor 3; JAKs: Janus kinases; LLC: Lewis lung carcinoma; IncRNAs: Long non-coding RNAs; LIF: Leukemia inhibitory factor; MDSCs: Myeloid-derived suppressor cells; MHC class II: Major histocompatibility complex class II; MMPs: Matrix metalloproteinases; MPLW515L: Somatic mutations at codon 515 of the thrombopoietin receptor; ncRNAs: Non-coding RNAs; $\mathrm{NH2}$ : Amino-terminal domain; NSCL C: Non-small cell lung cancer; NF-kB: Nuclear factor-kB; NTD: N-terminal domain; PC: Pancreatic cancer; PCa: Prostate cancer; PD-1: Programmed cell death protein 1; PDGFR: Platelet-derived growth factor receptor; PDL1: Programmed cell death 1 ligand 1; PKC $3 \|$ : Protein kinase C $\beta$ II; PMF: Primary myelofibrosis; PIAS: Protein inhibitor of activated STAT; PIGF: Placenta growth factor; PI3K: Phosphatidylinositol 3 kinase; Post-ET MF: Post-essential thrombocythemia myelofibrosis; Post-PV: Postpolycythemia vera; PROTAC: Proteolysis-targeting chimera; PTPases: Protein tyrosine phosphatases; PTPN1: Protein tyrosine phosphatase non-receptor type 1; PTPRD: Protein tyrosine phosphatase receptor type D; PTPRT: Protein tyrosine phosphatase receptor type T; RTK: Receptor tyrosine kinase; $\mathrm{SH}$ 2: Src homology 2 domain; SHP: Src homology 2 domain containing protein tyrosine phosphatase; SIRT1: Sirtuin 1; SMYD2: SET (Suppressor of variegation, Enhancer of Zeste, Trithorax) and MYND (Myeloid-Nervy-DEAF1) domain containing 2; SOCS: Suppressor of cytokine signaling; STAT: Signal transducer and activator of transcription; STING: Stimulator of interferon genes; STINGNPs: STING-activating nanoparticles; SUMO2/3: Small ubiquitin-like modifier 2/3; TAD: Transactivation domain; TBK-1: TANK-binding kinase 1; TCR: T cell receptor; TGF $\beta$ : Transforming growth factor $\beta$; Th1: T helper 1; TLR: Toll-like receptor; TME: Tumor microenvironment; TNFa: Tumor necrosis factor $\alpha$; Treg: Regulatory T-cell; VEGF: Vascular endothelial growth factor; VEGF R: Vascular endothelial growth factor receptor; 3'-UTR: 3'-untranslated region; 5-FU: 5-fluorouracil

\section{Acknowledgements}

None.

\section{Authors' contributions}

Y.T. and X.F. conceived the idea; S.Z., Q.T. and B.L. performed the literature search and draft the manuscript; W.H. revised and edited the manuscript; X.F. and Y.T. supervised and revised the manuscript. The author(s) read and approved the final manuscript. 


\section{Funding}

This work was supported by the Ministry of Science and Technology of China (2018ZX09201018-005), the National Natural Science Foundation of China $(81970561,81502631,81570527,91540113)$, the 1.3 .5 Project for Disciplines of Excellence, West China Hospital, Sichuan University (ZYJC18049), and National Clinical Research Center for Geriatrics, West China Hospital, Sichuan University (Z20191005, Z20201003).

\section{Availability of data and materials}

Not applicable.

\section{Ethics approval and consent to participate}

Not applicable.

\section{Consent for publication}

All the authors have approved the final manuscript for publication.

\section{Competing interests}

The authors declare that they have no competing interests.

\section{Author details}

${ }^{1}$ Division of Endocrinology and Metabolism, National Clinical Research Center for Geriatrics, State Key Laboratory of Biotherapy and Cancer Center, West China Hospital, Sichuan University and Collaborative Innovation Center of Biotherapy, Chengdu 610041, Sichuan, China. ${ }^{2}$ College of Life Sciences, Sichuan University, Chengdu 610041, Sichuan, China. ${ }^{3}$ Department of Integrated Traditional Chinese and Western Medicine, Sichuan Provincial Pancreatitis Centre and West China-Liverpool Biomedical Research Centre, West China Hospital, Sichuan University, Chengdu 610041, China.

\section{Received: 20 May 2020 Accepted: 4 September 2020}

\section{Published online: 24 September 2020}

\section{References}

1. Christofi T, Baritaki S, Falzone L, Libra M, Zaravinos A. Current Perspectives in Cancer Immunotherapy. Cancers (Basel). 2019;11

2. Hodi FS, O'Day SJ, McDermott DF, Weber RW, Sosman JA, Haanen JB, et al. Improved survival with ipilimumab in patients with metastatic melanoma. N Engl J Med. 2010;363:711-23.

3. Brahmer JR, Tykodi SS, Chow LQ, Hwu WJ, Topalian SL, Hwu P, et al. Safety and activity of anti-PD-L1 antibody in patients with advanced cancer. $\mathrm{N}$ Engl J Med. 2012;366:2455-65.

4. Topalian SL, Hodi FS, Brahmer JR, Gettinger SN, Smith DC, McDermott DF, et al. Safety, activity, and immune correlates of anti-PD-1 antibody in cancer. N Engl J Med. 2012;366:2443-54.

5. Darnell JE Jr, Kerr IM, Stark GR. Jak-STAT pathways and transcriptional activation in response to IFNs and other extracellular signaling proteins. Science. 1994;264:1415-21.

6. Hanlon MM, Rakovich T, Cunningham CC, Ansboro S, Veale DJ, Fearon U, et al. STAT3 Mediates the Differential Effects of Oncostatin M and TNFalpha on RA Synovial Fibroblast and Endothelial Cell Function. Front Immunol. 2019;10:2056

7. Rawlings JS, Rosler KM, Harrison DA. The JAKJSTAT signaling pathway. J Cell Sci. 2004;117:1281-3.

8. Johnson DE, O'Keefe RA, Grandis JR. Targeting the IL-6/JAK/STAT3 signalling axis in cancer. Nat Rev Clin Oncol. 2018;15:234-48.

9. Ishibashi K, Koguchi T, Matsuoka K, Onagi A, Tanji R, Takinami-Honda R, et al. Interleukin-6 induces drug resistance in renal cell carcinoma. Fukushima J Med Sci. 2018;64:103-10

10. Priego N, Zhu L, Monteiro C, Mulders M, Wasilewski D, Bindeman W, et al. STAT3 labels a subpopulation of reactive astrocytes required for brain metastasis. Nat Med. 2018;24:1024-35.

11. Bromberg JF, Wrzeszczynska MH, Devgan G, Zhao Y, Pestell RG, Albanese C, et al. Stat3 as an oncogene. Cell. 1999;98:295-303.

12. Wang T, Fahrmann JF, Lee H, Li YJ, Tripathi SC, Yue C, et al. JAK/STAT3Regulated Fatty Acid beta-Oxidation Is Critical for Breast Cancer Stem Cell Self-Renewal and Chemoresistance. Cell Metab. 2018:27:136-50 e5.

13. Kortylewski M, Kujawski M, Wang T, Wei S, Zhang S, Pilon-Thomas S, et al. Inhibiting Stat3 signaling in the hematopoietic system elicits multicomponent antitumor immunity. Nat Med. 2005;11:1314-21.
14. Villarino AV, Kanno Y, O'Shea JJ. Mechanisms and consequences of Jak-STAT signaling in the immune system. Nat Immunol. 2017;18:374-84.

15. Sgrignani J, Garofalo M, Matkovic M, Merulla J, Catapano CV, Cavalli A. Structural Biology of STAT3 and Its Implications for Anticancer Therapies Development. Int J Mol Sci. 2018;19.

16. Nadiminty N, Lou W, Lee SO, Lin X, Trump DL, Gao AC. Stat3 activation of NF-\{kappa\} B p100 processing involves CBP/p300-mediated acetylation. Proc Natl Acad Sci U S A. 2006;103:7264-9.

17. Gambi G, Di Simone E, Basso V, Ricci L, Wang R, Verma A, et al. The Transcriptional Regulator Sin3A Contributes to the Oncogenic Potential of STAT3. Cancer Res. 2019:79:3076-87.

18. Nie Y, Erion DM, Yuan Z, Dietrich M, Shulman Gl, Horvath TL, et al. STAT3 inhibition of gluconeogenesis is downregulated by SirT1. Nat Cell Biol. 2009; 11:492-500

19. Ma L, Huang C, Wang XJ, Xin DE, Wang LS, Zou QC, et al. Lysyl Oxidase 3 is a Dual-Specificity Enzyme Involved in STAT3 Deacetylation and Deacetylimination Modulation. Mol Cell. 2017;65:296-309.

20. Kim E, Kim M, Woo DH, Shin Y, Shin J, Chang N, et al. Phosphorylation of EZH2 activates STAT3 signaling via STAT3 methylation and promotes tumorigenicity of glioblastoma stem-like cells. Cancer Cell. 2013;23:839-52.

21. Yang J, Huang J, Dasgupta M, Sears N, Miyagi M, Wang B, et al. Reversible methylation of promoter-bound STAT3 by histone-modifying enzymes. Proc Natl Acad Sci U S A. 2010;107:21499-504.

22. Li LX, Fan LX, Zhou JX, Grantham JJ, Calvet JP, Sage J, et al. Lysine methyltransferase SMYD2 promotes cyst growth in autosomal dominant polycystic kidney disease. J Clin Invest. 2017;127:2751-64.

23. Zhou Z, Wang M, Li J, Xiao M, Chin YE, Cheng J, et al. SUMOylation and SENP3 regulate STAT3 activation in head and neck cancer. Oncogene. 2016; 35:5826-38.

24. Lin W, Luo J, Sun Y, Lin C, Li G, Niu Y, et al. ASC-J9((R)) suppresses prostate cancer cell invasion via altering the sumoylation-phosphorylation of STAT3. Cancer Lett. 2018:425:21-30.

25. Zhang L, Li J, Wang Q, Meng G, Lv X, Zhou H, et al. The relationship between microRNAs and the STAT3-related signaling pathway in cancer. Tumour Biol. 2017;39:1010428317719869.

26. Huan $\mathrm{L}$, Liang $\mathrm{LH}$, He $\mathrm{XH}$. Role of microRNAs in inflammation-associated liver cancer. Cancer Biol Med. 2016;13:407-25.

27. Guo R, Wu Z, Wang J, Li Q, Shen S, Wang W, et al. Development of a NonCoding-RNA-based EMT/CSC Inhibitory Nanomedicine for In Vivo Treatment and Monitoring of HCC. Adv Sci (Weinh). 2019:6:1801885.

28. Yang Y, Ding L, Hu Q, Xia J, Sun J, Wang X, et al. MicroRNA-218 functions as a tumor suppressor in lung cancer by targeting IL-6/STAT3 and negatively correlates with poor prognosis. Mol Cancer. 2017;16:141.

29. Zhang X, Sai B, Wang F, Wang L, Wang Y, Zheng L, et al. Hypoxic BMSCderived exosomal miRNAs promote metastasis of lung cancer cells via STAT3-induced EMT. Mol Cancer. 2019;18:40

30. Jin S, Yang X, Li J, Yang W, Ma H, Zhang Z. p53-targeted lincRNA-p21 acts as a tumor suppressor by inhibiting JAK2/STAT3 signaling pathways in head and neck squamous cell carcinoma. Mol Cancer. 2019:18:38.

31. Luo Z, Cao P. Long noncoding RNA PVT1 promotes hepatoblastoma cell proliferation through activating STAT3. Cancer Manag Res. 2019;11:8517-27.

32. Wang S, Liang K, Hu Q, Li P, Song J, Yang Y, et al. JAK2-binding long noncoding RNA promotes breast cancer brain metastasis. J Clin Invest. 2017;127:4498-515.

33. Liang C, Zhao T, Li H, He F, Zhao X, Zhang Y, et al. Long Non-coding RNA ITIH4-AS1 Accelerates the Proliferation and Metastasis of Colorectal Cancer by Activating JAK/STAT3 Signaling. Mol Ther Nucleic Acids. 2019;18:183-93.

34. Pichler M, Rodriguez-Aguayo C, Nam SY, Dragomir MP, Bayraktar R, Anfossi $S$, et al. Therapeutic potential of FLANC, a novel primate-specific long noncoding RNA in colorectal cancer. Gut. 2020.

35. Wang $Y$, Wu S, Zhu X, Zhang L, Deng J, Li F, et al. LncRNA-encoded polypeptide ASRPS inhibits triple-negative breast cancer angiogenesis. J Exp Med. 2020;217.

36. Hu D, Zhang Y. Circular RNA HIPK3 promotes glioma progression by binding to miR-124-3p. Gene. 2019;690:81-9.

37. Dong Y, Xu T, Zhong S, Wang B, Zhang H, Wang X, et al. Circ_0076305 regulates cisplatin resistance of non-small cell lung cancer via positively modulating STAT3 by sponging miR-296-5p. Life Sci. 2019;239:116984.

38. Liu Y, Song J, Liu Y, Zhou Z, Wang X. Transcription activation of circ-STAT3 induced by Gli2 promotes the progression of hepatoblastoma via acting as 
a sponge for miR-29a/b/c-3p to upregulate STAT3/Gli2. J Exp Clin Cancer Res. 2020;39:101.

39. Iliopoulos D, Jaeger SA, Hirsch HA, Bulyk ML, Struhl K. STAT3 activation of miR-21 and miR-181b-1 via PTEN and CYLD are part of the epigenetic switch linking inflammation to cancer. Mol Cell. 2010;39:493-506.

40. Loffler D, Brocke-Heidrich K, Pfeifer G, Stocsits C, Hackermuller J, Kretzschmar AK, et al. Interleukin-6 dependent survival of multiple myeloma cells involves the Stat3-mediated induction of microRNA-21 through a highly conserved enhancer. Blood. 2007;110:1330-3.

41. Gao H, Liu R, Sun X. STAT3-induced upregulation of IncRNA SNHG17 predicts a poor prognosis of melanoma and promotes cell proliferation and metastasis through regulating PI3K-AKT pathway. Eur Rev Med Pharmacol Sci. 2019;23:8000-10.

42. Du C, Wang HX, Chen P, Chen CH. STAT3-induced upregulation of IncRNA DUXAP8 functions as ceRNA for miR-577 to promote the migration and invasion in colorectal cancer through the regulation of RAB14. Eur Rev Med Pharmacol Sci. 2019;23:6105-18.

43. Chen JF, Wu P, Xia R, Yang J, Huo XY, Gu DY, et al. STAT3-induced IncRNA HAGLROS overexpression contributes to the malignant progression of gastric cancer cells via mTOR signal-mediated inhibition of autophagy. Mol Cancer. 2018;17:6.

44. Yu H, Pardoll D, Jove R. STATs in cancer inflammation and immunity: a leading role for STAT3. Nat Rev Cancer. 2009;9:798-809.

45. Pearce OMT, Delaine-Smith RM, Maniati E, Nichols S, Wang J, Bohm S, et al. Deconstruction of a Metastatic Tumor Microenvironment Reveals a Common Matrix Response in Human Cancers. Cancer Discov. 2018;8:304-19.

46. Azizi E, Carr AJ, Plitas G, Cornish AE, Konopacki C, Prabhakaran S, et al. Single-Cell Map of Diverse Immune Phenotypes in the Breast Tumor Microenvironment. Cell. 2018;174:1293-308 e36.

47. Martin JD, Cabral H, Stylianopoulos T, Jain RK. Improving cancer immunotherapy using nanomedicines: progress, opportunities and challenges. Nat Rev Clin Oncol. 2020.

48. Phuengkham H, Ren L, Shin IW, Lim YT. Nanoengineered Immune Niches for Reprogramming the Immunosuppressive Tumor Microenvironment and Enhancing Cancer Immunotherapy. Adv Mater. 2019;31:e1803322.

49. Herrmann A, Kortylewski M, Kujawski M, Zhang C, Reckamp K, Armstrong B, et al. Targeting Stat3 in the myeloid compartment drastically improves the in vivo antitumor functions of adoptively transferred T cells. Cancer Res. 2010;70:7455-64.

50. Iwata-Kajihara T, Sumimoto H, Kawamura N, Ueda R, Takahashi T, Mizuquchi $H$, et al. Enhanced cancer immunotherapy using STAT3-depleted dendritic cells with high Th1-inducing ability and resistance to cancer cell-derived inhibitory factors. J Immunol. 2011;187:27-36.

51. Siegel AM, Heimall J, Freeman AF, Hsu AP, Brittain E, Brenchley JM, et al. A critical role for STAT3 transcription factor signaling in the development and maintenance of human T cell memory. Immunity. 2011;35:806-18.

52. Gotthardt D, Putz EM, Straka E, Kudweis P, Biaggio M, Poli V, et al. Loss of STAT3 in murine NK cells enhances NK cell-dependent tumor surveillance. Blood. 2014;124:2370-9.

53. Wang T, Niu G, Kortylewski M, Burdelya L, Shain K, Zhang S, et al. Regulation of the innate and adaptive immune responses by Stat-3 signaling in tumor cells. Nat Med. 2004;10:48-54.

54. Icardi L, Lievens S, Mori R, Piessevaux J, De Cauwer L, De Bosscher K, et al. Opposed regulation of type I IFN-induced STAT3 and ISGF3 transcriptional activities by histone deacetylases (HDACS) 1 and 2. FASEB J. 2012;26:240-9.

55. Tsai MH, Lee CK. STAT3 Cooperates With Phospholipid Scramblase 2 to Suppress Type I Interferon Response. Front Immunol. 2018;9:1886.

56. Fan Y, Mao R, Yang J. NF-kappaB and STAT3 signaling pathways collaboratively link inflammation to cancer. Protein Cell. 2013;4:176-85.

57. Taniguchi K, Karin M. NF-kappaB, inflammation, immunity and cancer: coming of age. Nat Rev Immunol. 2018;18:309-24.

58. Lee H, Herrmann A, Deng JH, Kujawski M, Niu G, Li Z, et al. Persistently activated Stat3 maintains constitutive NF-kappaB activity in tumors. Cancer Cell. 2009;15:283-93.

59. Garg B, Giri B, Modi S, Sethi V, Castro I, Umland O, et al. NFkappaB in Pancreatic Stellate Cells Reduces Infiltration of Tumors by Cytotoxic T Cells and Killing of Cancer Cells, via Up-regulation of CXCL12. Gastroenterology. 2018;155:880-91 e8.

60. Saha A, Ahn S, Blando J, Su F, Kolonin MG, DiGiovanni J. Proinflammatory CXCL12-CXCR4/CXCR7 Signaling Axis Drives Myc-Induced Prostate Cancer in Obese Mice. Cancer Res. 2017;77:5158-68.
61. Shaim H, Estrov Z, Harris D, Hernandez Sanabria M, Liu Z, Ruvolo P, et al. The CXCR4-STAT3-IL-10 Pathway Controls the Immunoregulatory Function of Chronic Lymphocytic Leukemia and Is Modulated by Lenalidomide. Front Immunol. 2017;8:1773.

62. Rebe C, Ghiringhelli F. STAT3, a Master Regulator of Anti-Tumor Immune Response. Cancers (Basel). 2019;11.

63. Huynh J, Chand A, Gough D, Ernst M. Therapeutically exploiting STAT3 activity in cancer - using tissue repair as a road map. Nat Rev Cancer. 2019; 19:82-96.

64. Yu H, Kortylewski M, Pardoll D. Crosstalk between cancer and immune cells: role of STAT3 in the tumour microenvironment. Nat Rev Immunol. 2007;7: $41-51$.

65. Yoo SA, Kim M, Kang MC, Kong JS, Kim KM, Lee S, et al. Placental growth factor regulates the generation of $\mathrm{TH} 17$ cells to link angiogenesis with autoimmunity. Nat Immunol. 2019;20:1348-59.

66. Lin F, Meng X, Guo Y, Cao W, Liu W, Xia Q, et al. Epigenetic initiation of the TH17 differentiation program is promoted by Cxxc finger protein 1. Sci Adv. 2019;5:eaax1608.

67. Mohammadpour H, MacDonald CR, Qiao G, Chen M, Dong B, Hylander BL et al. beta2 Adrenergic receptor-mediated signaling regulates the immunosuppressive potential of myeloid-derived suppressor cells. J Clin Invest. 2019.

68. Takenaka MC, Gabriely G, Rothhammer V, Mascanfroni ID, Wheeler MA, Chao CC, et al. Control of tumor-associated macrophages and T cells in glioblastoma via AHR and CD39. Nat Neurosci. 2019;22:729-40.

69. Oweida AJ, Darragh L, Phan A, Binder D, Bhatia S, Mueller A, et al. STAT3 Modulation of Regulatory T Cells in Response to Radiation Therapy in Head and Neck Cancer. J Natl Cancer Inst. 2019.

70. Chen X, Song E. Turning foes to friends: targeting cancer-associated fibroblasts. Nat Rev Drug Discov. 2019;18:99-115.

71. Albrengues J, Bertero T, Grasset E, Bonan S, Maiel M, Bourget I, et al. Epigenetic switch drives the conversion of fibroblasts into proinvasive cancer-associated fibroblasts. Nat Commun. 2015;6:10204.

72. Yang $X$, Lin Y, Shi Y, Li B, Liu W, Yin W, et al. FAP Promotes Immunosuppression by Cancer-Associated Fibroblasts in the Tumor Microenvironment via STAT3-CCL2 Signaling. Cancer Res. 2016;76:4124-35.

73. Li X, Xu Q, Wu Y, Li J, Tang D, Han L, et al. A CCL2/ROS autoregulation loop is critical for cancer-associated fibroblasts-enhanced tumor growth of oral squamous cell carcinoma. Carcinogenesis. 2014;35:1362-70.

74. Heichler C, Scheibe K, Schmied A, Geppert Cl, Schmid B, Wirtz S, et al. STAT3 activation through IL-6/L-11 in cancer-associated fibroblasts promotes colorectal tumour development and correlates with poor prognosis. Gut. 2019.

75. Giovanelli P, Sandoval TA, Cubillos-Ruiz JR. Dendritic Cell Metabolism and Function in Tumors. Trends Immunol. 2019:40:699-718.

76. Farren MR, Carlson LM, Netherby CS, Lindner I, Li PK, Gabrilovich DI, et al. Tumor-induced STAT3 signaling in myeloid cells impairs dendritic cell generation by decreasing PKCbetall abundance. Sci Signal. 2014;7:ra16.

77. Calon A, Espinet E, Palomo-Ponce S, Tauriello DV, Iglesias M, Cespedes MV, et al. Dependency of colorectal cancer on a TGF-beta-driven program in stromal cells for metastasis initiation. Cancer Cell. 2012;22:571-84.

78. Ohlund D, Handly-Santana A, Biffi G, Elyada E, Almeida AS, Ponz-Sarvise M, et al. Distinct populations of inflammatory fibroblasts and myofibroblasts in pancreatic cancer. J Exp Med. 2017;214:579-96.

79. Huang H, Zhang Y, Gallegos V, Sorrelle N, Zaid MM, Toombs J, et al. Targeting TGFbetaR2-mutant tumors exposes vulnerabilities to stromal TGFbeta blockade in pancreatic cancer. EMBO Mol Med. 2019:e10515.

80. Ham IH, Oh HJ, Jin H, Bae CA, Jeon SM, Choi KS, et al. Targeting interleukin6 as a strategy to overcome stroma-induced resistance to chemotherapy in gastric cancer. Mol Cancer. 2019;18:68.

81. Lin $Q$, Ren L, Jian M, Xu P, Li J, Zheng P, et al. The mechanism of the premetastatic niche facilitating colorectal cancer liver metastasis generated from myeloid-derived suppressor cells induced by the S1PR1-STAT3 signaling pathway. Cell Death Dis. 2019;10:693.

82. Cheng JT, Deng YN, Yi HM, Wang GY, Fu BS, Chen WJ, et al. Hepatic carcinoma-associated fibroblasts induce IDO-producing regulatory dendritic cells through IL-6-mediated STAT3 activation. Oncogenesis. 2016;5:e198.

83. Nagathihalli NS, Castellanos JA, Shi C, Beesetty Y, Reyzer ML, Caprioli R, et al. Signal Transducer and Activator of Transcription 3, Mediated Remodeling of the Tumor Microenvironment Results in Enhanced Tumor Drug Delivery in a Mouse Model of Pancreatic Cancer. Gastroenterology. 2015;149:1932-43 e9. 
84. Sanz-Moreno V, Gaggioli C, Yeo M, Albrengues J, Wallberg F, Viros A, et al. ROCK and JAK1 signaling cooperate to control actomyosin contractility in tumor cells and stroma. Cancer Cell. 2011;20:229-45.

85. Gelain A, Mori M, Meneghetti F, Villa S. Signal Transducer and Activator of Transcription Protein 3 (STAT3): An Update on its Direct Inhibitors as Promising Anticancer Agents. Curr Med Chem. 2019;26:5165-206.

86. Beebe JD, Liu JY, Zhang JT. Two decades of research in discovery of anticancer drugs targeting STAT3, how close are we? Pharmacol Ther. 2018; 191:74-91.

87. Nagel-Wolfrum K, Buerger C, Wittig I, Butz K, Hoppe-Seyler F, Groner B. The interaction of specific peptide aptamers with the DNA binding domain and the dimerization domain of the transcription factor Stat3 inhibits transactivation and induces apoptosis in tumor cells. Mol Cancer Res. 2004; 2:170-82.

88. Miccoli A, Dhiani BA, Mehellou Y. Phosphotyrosine prodrugs: design, synthesis and anti-STAT3 activity of ISS-610 aryloxy triester phosphoramidate prodrugs. Medchemcomm. 2019;10:200-8.

89. Turkson J, Ryan D, Kim JS, Zhang Y, Chen Z, Haura E, et al. Phosphotyrosyl peptides block Stat3-mediated DNA binding activity, gene regulation, and cell transformation. J Biol Chem. 2001;276:45443-55.

90. Ji P, Xu X, Ma S, Fan J, Zhou Q, Mao X, et al. Novel 2-Carbonylbenzo [b] thiophene 1,1-Dioxide Derivatives as Potent Inhibitors of STAT3 Signaling Pathway. ACS Med Chem Lett. 2015;6:1010-4.

91. Lin L, Hutzen B, Zuo M, Ball S, Deangelis S, Foust E, et al. Novel STAT3 phosphorylation inhibitors exhibit potent growth-suppressive activity in pancreatic and breast cancer cells. Cancer Res. 2010;70:2445-54.

92. Li Z, Zhu T, Xu Y, Wu C, Chen J, Ren Y, et al. A novel STAT3 inhibitor, HJC0152, exerts potent antitumor activity in glioblastoma. Am J Cancer Res. 2019;9:699-713.

93. Liu Z, Wang H, Guan L, Lai C, Yu W, Lai M. LL1, a novel and highly selective STAT3 inhibitor, displays anti-colorectal cancer activities in vitro and in vivo. Br J Pharmacol. 2020;177:298-313.

94. Fuh B, Sobo M, Cen L, Josiah D, Hutzen B, Cisek K, et al. LLL-3 inhibits STAT3 activity, suppresses glioblastoma cell growth and prolongs survival in a mouse glioblastoma model. Br J Cancer. 2009;100:106-12.

95. Zuo M, Li C, Lin J, Javle M. LLL12, a novel small inhibitor targeting STAT3 for hepatocellular carcinoma therapy. Oncotarget. 2015;6:10940-9.

96. Wang H, Liu Z, Guan L, Li J, Chen S, Yu W, et al. LYW-6, a novel cryptotanshinone derived STAT3 targeting inhibitor, suppresses colorectal cancer growth and metastasis. Pharmacol Res. 2020;153:104661.

97. Kim LH, Khadka S, Shin JA, Jung JY, Ryu MH, Yu HJ, et al. Nitidine chloride acts as an apoptosis inducer in human oral cancer cells and a nude mouse xenograft model via inhibition of STAT3. Oncotarget. 2017; 8:91306-15.

98. Bai L, Zhou H, Xu R, Zhao Y, Chinnaswamy K, McEachern D, et al. A Potent and Selective Small-Molecule Degrader of STAT3 Achieves Complete Tumor Regression In Vivo. Cancer Cell. 2019;36:498-511 e17.

99. Schust J, Sperl B, Hollis A, Mayer TU, Berg T. Stattic: a small-molecule inhibitor of STAT3 activation and dimerization. Chem Biol. 2006;13:1235-42.

100. Akiyama Y, Nonomura C, Ashizawa T, lizuka A, Kondou R, Miyata H, et al. The anti-tumor activity of the STAT3 inhibitor STX-0119 occurs via promotion of tumor-infiltrating lymphocyte accumulation in temozolomideresistant glioblastoma cell line. Immunol Lett. 2017;190:20-5.

101. Soleimani AH, Garg SM, Paiva IM, Vakili MR, Alshareef A, Huang YH, et al. Micellar nano-carriers for the delivery of STAT3 dimerization inhibitors to melanoma. Drug Deliv Transl Res. 2017;7:571-81.

102. Siddiquee K, Zhang S, Guida WC, Blaskovich MA, Greedy B, Lawrence HR, et al. Selective chemical probe inhibitor of Stat3, identified through structure-based virtual screening, induces antitumor activity. Proc Natl Acad Sci U S A. 2007;104:7391-6.

103. Turkson J, Zhang S, Palmer J, Kay H, Stanko J, Mora LB, et al. Inhibition of constitutive signal transducer and activator of transcription 3 activation by novel platinum complexes with potent antitumor activity. Mol Cancer Ther. 2004;3:1533-42.

104. Buettner R, Corzano R, Rashid R, Lin J, Senthil M, Hedvat M, et al. Alkylation of cysteine 468 in Stat3 defines a novel site for therapeutic development. ACS Chem Biol. 2011;6:432-43.

105. Escobar Z, Bjartell A, Canesin G, Evans-Axelsson S, Sterner O, Hellsten R, et al. Preclinical Characterization of 3beta-(N-Acetyl I-cysteine methyl ester)-2abeta,3dihydrogaliellalactone (GPA512), a Prodrug of a Direct STAT3 Inhibitor for the Treatment of Prostate Cancer. J Med Chem. 2016;59:4551-62.
106. Son DJ, Zheng J, Jung YY, Hwang CJ, Lee HP, Woo JR, et al. MMPP Attenuates Non-Small Cell Lung Cancer Growth by Inhibiting the STAT3 DNA-Binding Activity via Direct Binding to the STAT3 DNA-Binding Domain. Theranostics. 2017;7:4632-42.

107. Huang W, Dong Z, Chen Y, Wang F, Wang CJ, Peng H, et al. Small-molecule inhibitors targeting the DNA-binding domain of STAT3 suppress tumor growth, metastasis and STAT3 target gene expression in vivo. Oncogene. 2016;35:783-92.

108. Souissi I, Ladam P, Cognet JA, Le Coquil S, Varin-Blank N, Baran-Marszak F, et al. A STAT3-inhibitory hairpin decoy oligodeoxynucleotide discriminates between STAT1 and STAT3 and induces death in a human colon carcinoma cell line. Mol Cancer. 2012;11:12.

109. Koppikar P, Abdel-Wahab O, Hedvat C, Marubayashi S, Patel J, Goel A, et al. Efficacy of the JAK2 inhibitor INCB16562 in a murine model of MPLW515Linduced thrombocytosis and myelofibrosis. Blood. 2010;115:2919-27.

110. Lo MC, Peterson LF, Yan M, Cong X, Hickman JH, Dekelver RC, et al. JAK inhibitors suppress $t(8 ; 21)$ fusion protein-induced leukemia. Leukemia. 2013; 27:2272-9.

111. Lu X, Zhang T, Zhu SJ, Xun Q, Tong L, Hu X, et al. Discovery of JND3229 as a New EGFR(C797S) Mutant Inhibitor with In Vivo Monodrug Efficacy. ACS Med Chem Lett. 2018;9:1123-7.

112. Holmstrom TH, Moilanen AM, Ikonen T, Bjorkman ML, Linnanen T, Wohlfahrt G, et al. ODM-203, a Selective Inhibitor of FGFR and VEGFR, Shows Strong Antitumor Activity, and Induces Antitumor Immunity. Mol Cancer Ther. 2019;18:28-38.

113. Pei J, Zhang Y, Luo Q, Zheng W, Li W, Zeng X, et al. STAT3 inhibition enhances CDN-induced STING signaling and antitumor immunity. Cancer Lett. 2019:450:110-22.

114. Lu C, Talukder A, Savage NM, Singh N, Liu K. JAK-STAT-mediated chronic inflammation impairs cytotoxic T lymphocyte activation to decrease antiPD-1 immunotherapy efficacy in pancreatic cancer. Oncoimmunology. 2017; 6:e1291106.

115. Yu GT, Mao L, Wu L, Deng WW, Bu LL, Liu JF, et al. Inhibition of SRC family kinases facilitates anti-CTLA4 immunotherapy in head and neck squamous cell carcinoma. Cell Mol Life Sci. 2018;75:4223-34.

116. Yang H, Lee WS, Kong SJ, Kim CG, Kim JH, Chang SK, et al. STING activation reprograms tumor vasculatures and synergizes with VEGFR2 blockade. J Clin Invest. 2019:130:4350-64.

117. Jonker DJ, Nott L, Yoshino T, Gill S, Shapiro J, Ohtsu A, et al. Napabucasin versus placebo in refractory advanced colorectal cancer: a randomised phase 3 trial. Lancet Gastroenterol Hepatol. 2018;3:263-70.

118. Reilley MJ, McCoon P, Cook C, Lyne P, Kurzrock R, Kim Y, et al. STAT3 antisense oligonucleotide AZD9150 in a subset of patients with heavily pretreated lymphoma: results of a phase $1 \mathrm{~b}$ trial. J Immunother Cancer. 2018;6:119.

119. Sen M, Thomas SM, Kim S, Yeh Jl, Ferris RL, Johnson JT, et al. First-in-human trial of a STAT3 decoy oligonucleotide in head and neck tumors: implications for cancer therapy. Cancer Discov. 2012;2:694-705.

120. Gupta V, Mesa RA, Deininger MW, Rivera CE, Sirhan S, Brachmann CB, et al. A phase $1 / 2$, open-label study evaluating twice-daily administration of momelotinib in myelofibrosis. Haematologica. 2017;102:94-102.

121. Berdeja J, Palandri F, Baer MR, Quick D, Kiladjian JJ, Martinelli G, et al. Phase 2 study of gandotinib (LY2784544) in patients with myeloproliferative neoplasms. Leuk Res. 2018;71:82-8.

122. Mascarenhas J, Hoffman R, Talpaz M, Gerds AT, Stein B, Gupta V, et al. Pacritinib vs Best Available Therapy, Including Ruxolitinib, in Patients With Myelofibrosis: A Randomized Clinical Trial. JAMA Oncol. 2018;4: 652-9.

123. Kim DW, Saussele S, Williams LA, Mohamed H, Rong Y, Zyczynski T, et al. Outcomes of switching to dasatinib after imatinib-related low-grade adverse events in patients with chronic myeloid leukemia in chronic phase: the DASPERSE study. Ann Hematol. 2018;97:1357-67.

124. Antonarakis ES, Heath El, Posadas EM, Yu EY, Harrison MR, Bruce JY, et al. A phase 2 study of KX2-391, an oral inhibitor of Src kinase and tubulin polymerization, in men with bone-metastatic castration-resistant prostate cancer. Cancer Chemother Pharmacol. 2013;71:883-92.

125. Finn RS, Qin S, Ikeda M, Galle PR, Ducreux M, Kim TY, et al. Atezolizumab plus Bevacizumab in Unresectable Hepatocellular Carcinoma. N Engl J Med. 2020;382:1894-905.

126. D'Angelo SP, Shoushtari AN, Keohan ML, Dickson MA, Gounder MM, Chi P, et al. Combined KIT and CTLA-4 Blockade in Patients with Refractory GIST 
and Other Advanced Sarcomas: A Phase Ib Study of Dasatinib plus Ipilimumab. Clin Cancer Res. 2017;23:2972-80.

127. Turkson J, Kim JS, Zhang S, Yuan J, Huang M, Glenn M, et al. Novel peptidomimetic inhibitors of signal transducer and activator of transcription 3 dimerization and biological activity. Mol Cancer Ther. 2004;3:261-9.

128. Lai AC, Crews CM. Induced protein degradation: an emerging drug discovery paradigm. Nat Rev Drug Discov. 2017;16:101-14.

129. Winter GE, Buckley DL, Paulk J, Roberts JM, Souza A, Dhe-Paganon S, et al. DRUG DEVELOPMENT. Phthalimide conjugation as a strategy for in vivo target protein degradation. Science. 2015;348:1376-81.

130. Lai AC, Toure M, Hellerschmied D, Salami J, Jaime-Figueroa S, Ko E, et al. Modular PROTAC Design for the Degradation of Oncogenic BCR-ABL. Angew Chem Int Ed Eng. 2016;55:807-10.

131. Burslem GM, Smith BE, Lai AC, Jaime-Figueroa S, McQuaid DC, Bondeson DP, et al. The Advantages of Targeted Protein Degradation Over Inhibition: An RTK Case Study. Cell Chem Biol. 2018;25:67-77 e3.

132. Khan S, Zhang X, Lv D, Zhang Q, He Y, Zhang P, et al. A selective BCL-XL PROTAC degrader achieves safe and potent antitumor activity. Nat Med. 2019:25:1938-47.

133. Njatcha C, Farooqui M, Kornberg A, Johnson DE, Grandis JR, Siegfried JM. STAT3 Cyclic Decoy Demonstrates Robust Antitumor Effects in Non-Small Cell Lung Cancer. Mol Cancer Ther. 2018;17:1917-26.

134. Hong D, Kurzrock R, Kim Y, Woessner R, Younes A, Nemunaitis J, et al. AZD9150, a next-generation antisense oligonucleotide inhibitor of STAT3 with early evidence of clinical activity in lymphoma and lung cancer. Sci Transl Med. 2015;7:314ra185.

135. Odate S, Veschi V, Yan S, Lam N, Woessner R, Thiele CJ. Inhibition of STAT3 with the Generation 2.5 Antisense Oligonucleotide, AZD9150, Decreases Neuroblastoma Tumorigenicity and Increases Chemosensitivity. Clin Cancer Res. 2017;23:1771-84.

136. Shastri A, Choudhary G, Teixeira M, Gordon-Mitchell S, Ramachandra N, Bernard L, et al. Antisense STAT3 inhibitor decreases viability of myelodysplastic and leukemic stem cells. J Clin Invest. 2018;128:5479-88.

137. Zhou J, Rossi J. Aptamers as targeted therapeutics: current potential and challenges. Nat Rev Drug Discov. 2017;16:181-202.

138. Esposito CL, Nuzzo S, Catuogno S, Romano S, de Nigris F, de Franciscis V. STAT3 Gene Silencing by Aptamer-siRNA Chimera as Selective Therapeutic for Glioblastoma. Mol Ther Nucleic Acids. 2018;10:398-411.

139. Yoon S, Wu X, Armstrong B, Habib N, Rossi JJ. An RNA Aptamer Targeting the Receptor Tyrosine Kinase PDGFRalpha Induces Anti-tumor Effects through STAT3 and p53 in Glioblastoma. Mol Ther Nucleic Acids. 2019;14:131-41.

140. Heppler LN, Frank DA. Targeting Oncogenic Transcription Factors: Therapeutic Implications of Endogenous STAT Inhibitors. Trends Cancer. 2017;3:816-27

141. Khan MW, Saadalla A, Ewida AH, Al-Katranji K, Al-Saoudi G, Giaccone ZT, et al. The STAT3 inhibitor pyrimethamine displays anti-cancer and immune stimulatory effects in murine models of breast cancer. Cancer Immunol Immunother. 2018;67:13-23.

142. Li H, Liu A, Zhao Z, Xu Y, Lin J, Jou D, et al. Fragment-based drug design and drug repositioning using multiple ligand simultaneous docking (MLSD): identifying celecoxib and template compounds as novel inhibitors of signal transducer and activator of transcription 3 (STAT3). J Med Chem. 2011;54: 5592-6.

143. Huang $R$, Jing $X$, Huang $X$, Pan $Y$, Fang $Y$, Liang G, et al. Bifunctional Naphthoquinone Aromatic Amide-Oxime Derivatives Exert Combined Immunotherapeutic and Antitumor Effects through Simultaneous Targeting of Indoleamine-2,3-dioxygenase and Signal Transducer and Activator of Transcription 3. J Med Chem. 2020.

144. Buchert M, Burns CJ, Ernst M. Targeting JAK kinase in solid tumors: emerging opportunities and challenges. Oncogene. 2016;35:939-51.

145. Kang S, Tanaka T, Narazaki M, Kishimoto T. Targeting Interleukin-6 Signaling in Clinic. Immunity. 2019;50:1007-23.

146. Liu L, Nam S, Tian Y, Yang F, Wu J, Wang Y, et al. 6-Bromoindirubin-3'-oxime inhibits JAK/STAT3 signaling and induces apoptosis of human melanoma cells. Cancer Res. 2011;71:3972-9.

147. Tian Y, Nam S, Liu L, Yakushijin F, Yakushijin K, Buettner R, et al. Spirooxindole derivative SOID-8 induces apoptosis associated with inhibition of JAK2/STAT3 signaling in melanoma cells. PLoS One. 2012;7:e49306.

148. Wen W, Wu J, Liu L, Tian Y, Buettner R, Hsieh MY, et al. Synergistic antitumor effect of combined inhibition of EGFR and JAK/STAT3 pathways in human ovarian cancer. Mol Cancer. 2015;14:100.
149. Zhang Q, Liu RX, Chan KW, Hu J, Zhang J, Wei L, et al. Exosomal transfer of p-STAT3 promotes acquired 5-FU resistance in colorectal cancer cells. J Exp Clin Cancer Res. 2019;38:320.

150. Kalluri R, LeBleu VS. The biology, function, and biomedical applications of exosomes. Science. 2020;367.

151. Xu H, Du X, Xu J, Zhang Y, Tian Y, Liu G, et al. Pancreatic beta cell microRNA-26a alleviates type 2 diabetes by improving peripheral insulin sensitivity and preserving beta cell function. PLoS Biol. 2020;18:e3000603.

152. June CH, O'Connor RS, Kawalekar OU, Ghassemi S, Milone MC. CAR T cell immunotherapy for human cancer. Science. 2018;359:1361-5.

153. Kennedy LB, Salama AKS. A review of cancer immunotherapy toxicity. CA Cancer J Clin. 2020.

154. O'Reilly EM, Oh DY, Dhani N, Renouf DJ, Lee MA, Sun W, et al. Durvalumab With or Without Tremelimumab for Patients With Metastatic Pancreatic Ductal Adenocarcinoma: A Phase 2 Randomized Clinical Trial. JAMA Oncol. 2019

155. Zhou Y, Fei M, Zhang G, Liang WC, Lin W, Wu Y, et al. Blockade of the Phagocytic Receptor MerTK on Tumor-Associated Macrophages Enhances P2X7R-Dependent STING Activation by Tumor-Derived cGAMP. Immunity. 2020;52:357-73 e9.

156. Hellmann MD, Paz-Ares L, Bernabe Caro R, Zurawski B, Kim SW, Carcereny Costa E, et al. Nivolumab plus Ipilimumab in Advanced Non-Small-Cell Lung Cancer. N Engl J Med. 2019;381:2020-31.

157. Atsaves V, Tsesmetzis N, Chioureas D, Kis L, Leventaki V, Drakos E, et al. PDL1 is commonly expressed and transcriptionally regulated by STAT3 and MYC in ALK-negative anaplastic large-cell lymphoma. Leukemia. 2017;31: 1633-7.

158. Song TL, Nairismagi ML, Laurensia Y, Lim JQ, Tan J, Li ZM, et al. Oncogenic activation of the STAT3 pathway drives PD-L1 expression in natural killer/Tcell lymphoma. Blood. 2018;132:1146-58.

159. Garcia-Diaz A, Shin DS, Moreno BH, Saco J, Escuin-Ordinas H, Rodriguez GA, et al. Interferon Receptor Signaling Pathways Regulating PD-L1 and PD-L2 Expression. Cell Rep. 2017;19:1189-201.

160. Yoyen-Ermis D, Tunali G, Tavukcuoglu E, Horzum U, Ozkazanc D, Sutlu T, et al. Myeloid maturation potentiates STAT3-mediated atypical IFN-gamma signaling and upregulation of PD-1 ligands in AML and MDS. Sci Rep. 2019. 9:11697.

161. Herrmann A, Lahtz C, Nagao T, Song JY, Chan WC, Lee H, et al. CTLA4 Promotes Tyk2-STAT3-Dependent B-cell Oncogenicity. Cancer Res. 2017;77: 5118-28.

162. Hsu P, Santner-Nanan B, Hu M, Skarratt K, Lee CH, Stormon M, et al. IL-10 Potentiates Differentiation of Human Induced Regulatory T Cells via STAT3 and Foxo1. J Immunol. 2015;195:3665-74.

163. Keremu A, Aimaiti A, Liang Z, Zou X. Role of the HDAC6/STAT3 pathway in regulating PD-L1 expression in osteosarcoma cell lines. Cancer Chemother Pharmacol. 2019;83:255-64.

164. Celada LJ, Kropski JA, Herazo-Maya JD, Luo W, Creecy A, Abad AT, et al. PD1 up-regulation on CD4(+) T cells promotes pulmonary fibrosis through STAT3-mediated IL-17A and TGF-beta1 production. Sci Transl Med. 2018;10.

165. Celada LJ, Rotsinger JE, Young A, Shaginurova G, Shelton D, Hawkins C, et al. Programmed Death-1 Inhibition of Phosphatidylinositol 3-Kinase/AKT/ Mechanistic Target of Rapamycin Signaling Impairs Sarcoidosis CD4(+) T Cell Proliferation. Am J Respir Cell Mol Biol. 2017:56:74-82.

166. Krasilnikov M, Ivanov VN, Dong J, Ronai Z. ERK and PI3K negatively regulate STAT-transcriptional activities in human melanoma cells: implications towards sensitization to apoptosis. Oncogene. 2003;22:4092-101.

167. Mace TA, Shakya R, Pitarresi JR, Swanson B, McQuinn CW, Loftus S, et al. IL-6 and PD-L1 antibody blockade combination therapy reduces tumour progression in murine models of pancreatic cancer. Gut. 2018;67:320-32.

168. Liu H, Shen J, Lu K. IL-6 and PD-L1 blockade combination inhibits hepatocellular carcinoma cancer development in mouse model. Biochem Biophys Res Commun. 2017:486:239-44.

169. Luo F, Luo M, Rong QX, Zhang H, Chen Z, Wang F, et al. Niclosamide, an antihelmintic drug, enhances efficacy of PD-1/PD-L1 immune checkpoint blockade in non-small cell lung cancer. J Immunother Cancer. 2019;7:245.

170. Bagley SJ, O'Rourke DM. Clinical investigation of CAR T cells for solid tumors: Lessons learned and future directions. Pharmacol Ther. 2019;107419.

171. Du H, Hirabayashi K, Ahn S, Kren NP, Montgomery SA, Wang X, et al. Antitumor Responses in the Absence of Toxicity in Solid Tumors by Targeting B7-H3 via Chimeric Antigen Receptor T Cells. Cancer Cell. 2019;35: 221-37 e8. 
172. Majzner RG, Theruvath JL, Nellan A, Heitzeneder S, Cui Y, Mount CW, et al. CAR T Cells Targeting B7-H3, a Pan-Cancer Antigen, Demonstrate Potent Preclinical Activity Against Pediatric Solid Tumors and Brain Tumors. Clin Cancer Res. 2019:25:2560-74.

173. Fraietta JA, Lacey SF, Orlando EJ, Pruteanu-Malinici I, Gohil M, Lundh S, et al. Determinants of response and resistance to CD19 chimeric antigen receptor (CAR) T cell therapy of chronic lymphocytic leukemia. Nat Med. 2018;24: $563-71$.

174. Kagoya Y, Tanaka S, Guo T, Anczurowski M, Wang CH, Saso K, et al. A novel chimeric antigen receptor containing a JAK-STAT signaling domain mediates superior antitumor effects. Nat Med. 2018;24:352-9.

175. Wang Y, Jiang H, Luo H, Sun Y, Shi B, Sun R, et al. An IL-4/21 Inverted Cytokine Receptor Improving CAR-T Cell Potency in Immunosuppressive Solid-Tumor Microenvironment. Front Immunol. 2019;10:1691.

176. Shah NN, Fry TJ. Mechanisms of resistance to CAR T cell therapy. Nat Rev Clin Oncol. 2019;16:372-85.

177. Guha P, Gardell J, Darpolor J, Cunetta M, Lima M, Miller G, et al. STAT3 inhibition induces Bax-dependent apoptosis in liver tumor myeloid-derived suppressor cells. Oncogene. 2019;38:533-48.

178. Corrales L, McWhirter SM, Dubensky TW Jr, Gajewski TF. The host STING pathway at the interface of cancer and immunity. J Clin Invest. 2016;126: 2404-11.

179. Ramanjulu JM, Pesiridis GS, Yang J, Concha N, Singhaus R, Zhang SY, et al. Design of amidobenzimidazole STING receptor agonists with systemic activity. Nature. 2018:564:439-43.

180. Shae D, Becker KW, Christov P, Yun DS, Lytton-Jean AKR, Sevimli S, et al. Endosomolytic polymersomes increase the activity of cyclic dinucleotide STING agonists to enhance cancer immunotherapy. Nat Nanotechnol. 2019; 14:269-78.

181. Zhu Q, Man SM, Gurung P, Liu Z, Vogel P, Lamkanfi M, et al. Cutting edge: STING mediates protection against colorectal tumorigenesis by governing the magnitude of intestinal inflammation. J Immunol. 2014;193:4779-82.

182. Zhang CX, Ye SB, Ni JJ, Cai TT, Liu YN, Huang DJ, et al. STING signaling remodels the tumor microenvironment by antagonizing myeloid-derived suppressor cell expansion. Cell Death Differ. 2019;26:2314-28.

183. Hsia HC, Hutti JE, Baldwin AS. Cytosolic DNA Promotes Signal Transducer and Activator of Transcription 3 (STAT3) Phosphorylation by TANK-binding Kinase 1 (TBK1) to Restrain STAT3 Activity. J Biol Chem. 2017;292:5405-17.

184. Moreira D, Adamus T, Zhao X, Su YL, Zhang Z, White SV, et al. STAT3 Inhibition Combined with CpG Immunostimulation Activates Antitumor Immunity to Eradicate Genetically Distinct Castration-Resistant Prostate Cancers. Clin Cancer Res. 2018;24:5948-62.

185. Nefedova Y, Nagaraj S, Rosenbauer A, Muro-Cacho C, Sebti SM, Gabrilovich DI. Regulation of dendritic cell differentiation and antitumor immune response in cancer by pharmacologic-selective inhibition of the janusactivated kinase 2/signal transducers and activators of transcription 3 pathway. Cancer Res. 2005;65:9525-35.

\section{Publisher's Note}

Springer Nature remains neutral with regard to jurisdictional claims in published maps and institutional affiliations.

\section{Ready to submit your research? Choose BMC and benefit from}

- fast, convenient online submission

- thorough peer review by experienced researchers in your field

- rapid publication on acceptance

- support for research data, including large and complex data types

- gold Open Access which fosters wider collaboration and increased citations

- maximum visibility for your research: over $100 \mathrm{M}$ website views per year

At $\mathrm{BMC}$, research is always in progress.

Learn more biomedcentral.com/submissions 